\title{
On a class of stochastic partial differential equations with multiple invariant measures
}

\author{
Bálint Farkas, Martin Friesen, Barbara Rüdiger(1) and \\ Dennis Schroers
}

\begin{abstract}
In this work we investigate the long-time behavior for Markov processes obtained as the unique mild solution to stochastic partial differential equations in a Hilbert space. We analyze the existence and characterization of invariant measures as well as convergence of transition probabilities. While in the existing literature typically uniqueness of invariant measures is studied, we focus on the case where the uniqueness of invariant measures fails to hold. Namely, introducing a generalized dissipativity condition combined with a decomposition of the Hilbert space, we prove the existence of multiple limiting distributions in dependence of the initial state of the process and study the convergence of transition probabilities in the Wasserstein 2-distance. Finally, we apply our results to Lévy driven Ornstein-Uhlenbeck processes, the Heath-Jarrow-MortonMusiela equation as well as to stochastic partial differential equations with delay.
\end{abstract}

Mathematics Subject Classification. Primary 37L40, 60J25; Secondary 37A30, $37 \mathrm{~L} 55$.

Keywords. Stochastic partial differential equations, Multiple invariant measures, Long time behaviour, Dissipativity condition, Semigroups, Projection operators, Wasserstein metrics, Yosida approximation, HeathJarrow-Morton-Musiela equation, Delay equations.

\section{Introduction}

Stochastic partial differential equations arise in the modelling of applications in mathematical physics (e.g. Navier-Stokes equations [9, 18,22,37] or stochastic non-linear Schrödinger equations $[4,13]$ ), biology (e.g. catalytic branching processes $[12,30])$, and finance (e.g. forward prices $[16,24,38])$. While the construction of solutions to the underlying stochastic equations is an important mathematical issue, having applications in mind it is indispensable to also 
study their specific properties. Among them, an investigation of the long-time behavior of solutions, that is existence and uniqueness of invariant measures and convergence of transition probabilities, are often important and at the same time also challenging mathematical topics. In this work we investigate the long-time behavior of mild solutions to the stochastic partial differential equation of the form

$$
d X_{t}=\left(A X_{t}+F\left(X_{t}\right)\right) d t+\sigma\left(X_{t}\right) d W_{t}+\int_{E} \gamma\left(X_{t}, \nu\right) \tilde{N}(d t, d \nu), \quad t \geq 0
$$

on a separable Hilbert space $H$, where $(A, D(A))$ is the generator of a strongly continuous semigroup $(S(t))_{t \geq 0}$ on $H,\left(W_{t}\right)_{t \geq 0}$ is a $Q$-Wiener process and $\widetilde{N}(d t, d \nu)$ denotes a compensated Poisson random measure with compensator $d t \mu(d \nu)$ on $\mathbb{R}_{+} \times E$ with $E$ a Polish space. The precise conditions need to be imposed on these objects will be formulated in the subsequent sections. We focus in particular on SPDEs with multiple limiting distributions.

In the literature the study on the existence and uniqueness of invariant measures often relies on different variants of a dissipativity condition. The simplest form of such a dissipativity condition is: There exists $\alpha>0$ such that

$$
\langle A x-A y, x-y\rangle_{H}+\langle F(x)-F(y), x-y\rangle_{H} \leq-\alpha\|x-y\|_{H}^{2}, \quad x, y \in D(A) .
$$

Indeed, if (1.2) is satisfied, $\sigma$ and $\gamma$ are globally Lipschitz-continuous, and $\alpha$ is large enough, then there exists a unique invariant measure for the Markov process obtained from (1.1), see, e.g., [32, Section 16], [10, Chapter 11, Section $5]$, and [36] where such a condition was formulated for the Yosida approximations of the operator $(A, D(A))$. Note that (1.2) is satisfied, if $F$ is globally Lipschitz continuous and $(A, D(A))$ satisfies for some $\beta>0$ large enough the inequality $\langle A x, x\rangle_{H} \leq-\beta\|x\|_{H}^{2}, x \in D(A)$, i.e. $(A, D(A))$ is the generator of a strongly continuous semigroup satisfying $\|S(t)\|_{L(H)} \leq \mathrm{e}^{-\beta t}$. Here and below we denote by $L(H)$ the space of bounded linear operators from $H$ to $H$ and by $\|\cdot\|_{L(H)}$ its operator norm. For weaker variants of the dissipativity condition (e.g. cases where (1.2) only holds for $\|x\|_{H},\|y\|_{H} \geq R$ for some $R>0$ ), in general one can neither guarantee the existence nor uniqueness of an invariant measure. Hence, to treat such cases, additional arguments, e.g. coupling methods, are required. Such arguments have been applied to different stochastic partial differential equations on Hilbert spaces in [33-35] where existence and, in particular, uniqueness of invariant measures was studied. We also mention $[7,23]$ for an extension of Harris-type theorems for Wasserstein distances, and $[21,25]$ for extensions of coupling methods.

In contrast to the aforementioned methods and applications, several stochastic models exhibit phase transition phenomena where uniqueness of invariant measures fails to hold. For instance, the generator $(A, D(A))$ and drift $F$ appearing in the Heath-Jarrow-Morton-Musiela equation do not satisfy (1.2), but instead $F$ is globally Lipschitz continuous and the semigroup generated by $(A, D(A))$ satisfies

$$
\|S(t) x-P x\|_{H} \leq \mathrm{e}^{-\alpha t}\|x-P x\|_{H}
$$


for some projection operator $P$. Based on this property it was shown in $[36,38]$ that the Heath-Jarrow-Morton-Musiela equation has infinitely many invariant measures parametrized by the initial state of the process, see also Sect. 6. Another example is related to stochastic Volterra equations as studied, e.g., in [6]. There, using a representation of stochastic Volterra equations via SPDEs and combined with some arguments originated from the study of the HeathJarrow-Morton-Musiela equation, the authors studied existence of limiting distributions allowing, in particular, that these distributions depend on the initial state of the process.

In this work we provide a general and unified approach for the study of multiple invariant measures and, moreover, we show that with dependence on the initial distribution the law of the mild solution of (1.1) is governed in the limit $t \rightarrow \infty$ by one of the invariant measures. In particular, we show that the methods developed in $[6,36,38]$ can be embedded as a special case of a general framework where one replaces (1.2) by a weaker dissipativity condition, which we call hereinafter generalized dissipativity condition:

(GDC) There exists a projection operator $P_{1}$ on the Hilbert space $H$ and there exist constants $\alpha>0, \beta \geq 0$ such that, for $x, y \in D(A)$, one has:

$$
\begin{gathered}
\langle A x-A y, x-y\rangle_{H}+\langle F(x)-F(y), x-y\rangle_{H} \\
\leq-\alpha\|x-y\|_{H}^{2}+(\alpha+\beta)\left\|P_{1} x-P_{1} y\right\|_{H}^{2} .
\end{gathered}
$$

Note that for the special case $P_{1}=0$ condition (GDC) contains the classical dissipativity condition. However, when $P_{1} \neq 0$, the additional term $\| P_{1} x-$ $P_{1} y \|_{H}^{2}$ describes the influence of the non-dissipative part of the drift. Sufficient conditions and additional remarks on this condition are collected in the end of Sect. 2 while particular examples are discussed in Sects. 5-6.

We will show that under condition (GDC) and additional restrictions on the projected coefficients $P_{1} F, P_{1} \sigma$, and $P_{1} \gamma$, the Markov process obtained from (1.1) has for each initial data $X_{0}=x$ a limiting distribution $\pi_{x}$ depending only on $P_{1} x$. on This will often imply that there are multiple limiting distributions for (1.1). Moreover, the transition probabilities converge exponentially fast in the Wasserstein 2-distance to this limiting distribution. In order to prove this result, we first decompose the Hilbert space $H$ according to

$$
H=H_{0} \oplus H_{1}, \quad x=P_{0} x+P_{1} x, \quad P_{0}:=I-P_{1},
$$

where $I$ denotes the identity operator on $H$, and then investigate the components $P_{0} X_{t}$ and $P_{1} X_{t}$ separately. Based on an technique from [39], we construct, for each $\tau \geq 0$, a coupling of $X_{t}$ and $X_{t+\tau}$. This coupling will be then used to efficiently estimate the Wasserstein 2-distance for the solution started at two different points.

This work is organized as follows. In Sect. 2 we first discuss the special case where $F, \sigma, \gamma$ are independent of $X$. In such a case $X$ is an OrnsteinUhlenbeck type process and the collection of invariant measures can be easily 
characterized by its characteristic function. This section can be seen as a motivation for our more general results discussed in the subsequent sections. More precisely, we could also have studied the Ornstein-Uhlenbeck process by our general results from Sect. 4, however, in such a case we need to impose unnecessary strong conditions on the Lévy measure and would not obtain the characterization of invariant measures in terms of their Fourier transforms. Afterward, we investigate in Sects. 3-5 the general case for which the methods from Sect. 2, that is convergence of the Fourier transform, can not be applied. More precisely, after having introduced and discussed in Sect. 3 the generalized dissipativity condition (GDC), we state the precise conditions imposed on the coefficients of the SPDE (1.1), discuss some properties of the solution and then provide sufficient conditions for the generalized dissipativity condition (GDC). Based on condition (GDC) we also derive an estimate on the trajectories of the process when started at two different initial points, i.e. we estimate the $L^{2}$-norm of $X_{t}^{x}-X_{t}^{y}$ when $x \neq y$. Based on this estimate, we then state and prove our main results in Sect. 4. Examples are then discussed in the subsequent Sects. 5 and 6. Namely, the Heath-Jarrow-Morton-Musiela equation is considered in Sect. 5 for which we first show that the main results of Sect. 4 contain $[36,38]$, and then extend these results by characterizing its multiple limiting distributions more explicitly. Finally, we apply our results in Sect. 6 to an SPDE with delay.

\section{Ornstein-Uhlenbeck process in a Hilbert space}

Let $H$ be a separable Hilbert space and let $\left(Z_{t}\right)_{t \geq 0}$ be an $H$-valued Lévy process with Lévy triplet $(b, Q, \mu)$ defined on a stochastic basis $\left(\Omega, \mathcal{F},\left(\mathcal{F}_{t}\right)_{t \geq 0}, \mathbb{P}\right)$ with the usual conditions. This has characteristic exponent $\Psi$ of Lévy-Khinchine form, i.e.

$$
\mathbb{E}\left[\mathrm{e}^{\mathrm{i}\left\langle u, Z_{t}\right\rangle_{H}}\right]=\mathrm{e}^{t \Psi(u)}, \quad u \in H, \quad t>0
$$

with $\Psi$ given by

$$
\Psi(u)=\mathrm{i}\langle b, u\rangle_{H}-\frac{1}{2}\langle Q u, u\rangle_{H}+\int_{H}\left(\mathrm{e}^{\mathrm{i}\langle u, z\rangle_{H}}-1-\mathrm{i}\langle u, z\rangle_{H} \mathbb{1}_{\left\{\|z\|_{H} \leq 1\right\}}\right) \mu(d z),
$$

where $b \in H$ denotes the drift, $Q$ denotes the covariance operator being a positive, symmetric, trace-class operator on $H$, and $\mu$ is a Lévy measure on $H$ (see e.g. $[3,27,28,32])$. Let $(S(t))_{t \geq 0}$ be a strongly continuous semigroup on $H$. The Ornstein-Uhlenbeck process driven by $\left(Z_{t}\right)_{t \geq 0}$ is the unique mild solution to

$$
d X_{t}^{x}=A X_{t}^{x} d t+d Z_{t}, \quad X_{0}^{x}=x \in H, \quad t \geq 0,
$$

where $(A, D(A))$ denotes the generator of $(S(t))_{t \geq 0}$, i.e. $\left(X_{t}^{x}\right)_{t \geq 0}$ satisfies

$$
X_{t}^{x}=S(t) x+\int_{0}^{t} S(t-s) d Z_{s}, \quad t \geq 0 .
$$


The characteristic function of $\left(X_{t}^{x}\right)_{t \geq 0}$ is given by

$$
\mathbb{E}\left[\mathrm{e}^{\mathrm{i}\left\langle u, X_{t}^{x}\right\rangle_{H}}\right]=\exp \left(\mathrm{i}\langle S(t) x, u\rangle_{H}+\int_{0}^{t} \Psi\left(S(r)^{*} u\right) d r\right), \quad u \in H, \quad t \geq 0 .
$$

See e.g. the review article [3] where also sufficient conditions for the existence and for the uniqueness as well as properties of invariant measures are discussed. It is well-known that the Ornstein-Uhlenbeck process has a unique invariant measure provided that $(S(t))_{t \geq 0}$ is uniformly exponentially stable, that is

$$
\exists \alpha>0, M \geq 1: \quad\|S(t)\|_{L(H)} \leq M \mathrm{e}^{-\alpha t}, \quad t \geq 0,
$$

and the Lévy measure $\mu$ satisfies a log-integrability condition for its big jumps

$$
\int_{\left\{\|z\|_{H}>1\right\}} \log \left(1+\|z\|_{H}\right) \mu(d z)<\infty .
$$

Below we show that for a uniformly convergent semigroup $(S(t))_{t \geq 0}$ the corresponding Ornstein-Uhlenbeck process may admit multiple invariant measures parameterized by the range of the limiting projection operator of the semigroup.

Theorem 2.1. Suppose that $(S(t))_{t \geq 0}$ is uniformly exponentially convergent, i.e. there exists a projection operator $P$ on $H$ and constants $M \geq 1, \alpha>0$ such that

$$
\|S(t) x-P x\|_{H} \leq M\|x\|_{H} \mathrm{e}^{-\alpha t}, \quad t \geq 0, x \in H .
$$

Suppose that the Lévy process satisfies the following conditions:

(i) The drift $b$ satisfies $P b=0$.

(ii) The covariance operator $Q$ satisfies $P Q u=0$ for all $u \in H$.

(iii) The Lévy measure $\mu$ is supported on $\operatorname{ker}(P)$ and satisfies (2.2).

Then for each $x \in H$ it holds

$$
X_{t}^{x} \longrightarrow P x+X_{\infty}^{0}, \quad t \rightarrow \infty
$$

in law, where $X_{\infty}^{0}$ is an $H$-valued random variable determined by

$$
\mathbb{E}\left[\mathrm{e}^{\mathrm{i}\left\langle u, X_{\infty}^{0}\right\rangle_{H}}\right]=\exp \left(\int_{0}^{\infty} \Psi\left(S(r)^{*} u\right) d r\right) .
$$

In particular, the set of all limiting distributions for the Ornstein-Uhlenbeck process $\left(X_{t}^{x}\right)_{t \geq 0}$ is given by $\left\{\delta_{y} * \mu_{\infty} \mid y \in \operatorname{ran}(P)\right\}$, where $\mu_{\infty}$ denotes the law of $X_{\infty}^{0}$.

Proof. We first prove the existence of a constant $C>0$ such that

$$
\int_{0}^{\infty}\left|\Psi\left(S(r)^{*} u\right)\right| d r \leq C\left(\|u\|_{H}+\|u\|_{H}^{2}\right), \quad u \in H
$$


where $S(r)^{*}$ denotes the adjoint operator to $S(r)$ on $L(H)$. To do so we estimate

$$
\begin{aligned}
\left|\Psi\left(S(r)^{*} u\right)\right| \leq & \left|\left\langle b, S(r)^{*} u\right\rangle_{H}\right|+\left|\left\langle Q S(r)^{*} u, S(r)^{*} u\right\rangle_{H}\right| \\
& +\int_{\left\{\|z\|_{H} \leq 1\right\}}\left|\mathrm{e}^{\mathrm{i}\left\langle S(r)^{*} u, z\right\rangle_{H}}-1-\mathrm{i}\left\langle S(r)^{*} u, z\right\rangle_{H}\right| \mu(d z) \\
& +\int_{\left\{\|z\|_{H}>1\right\}}\left|\mathrm{e}^{\mathrm{i}\left\langle S(r)^{*} u, z\right\rangle_{H}}-1\right| \mu(d z) \\
= & I_{1}+I_{2}+I_{3}+I_{4} .
\end{aligned}
$$

We find by (2.3) that $\|S(r) x\|_{H} \leq M \mathrm{e}^{-\alpha r}\|x\|_{H}$ for all $x \in \operatorname{ker}(P)$ and hence

$$
I_{1}=\left|\langle S(r) b, u\rangle_{H}\right| \leq\|u\|_{H}\|S(r) b\|_{H} \leq\|u\|_{H} M \mathrm{e}^{-\alpha r}\|b\|_{H} .
$$

For the second term $I_{2}$ we use $\operatorname{ran}(Q) \subset \operatorname{ker}(P)$ so that

$$
\|S(r) Q u\|_{H} \leq M \mathrm{e}^{-\alpha r}\|Q u\|_{H} \leq \mathrm{e}^{-\alpha r}\|Q\|_{L(H)}\|u\|_{H} .
$$

This yields $\left\|Q S(r)^{*}\right\|_{L(H)}=\|S(r) Q\|_{L(H)} \leq M \mathrm{e}^{-\alpha r}\|Q\|_{L(H)}$ and hence

$$
\begin{aligned}
I_{2} & =\left|\left\langle Q S(r)^{*} u, S(r)^{*} u\right\rangle_{H}\right| \\
& \leq\left\|Q S(r)^{*} u\right\|_{H}\left\|S(r)^{*} u\right\|_{H} \\
& \leq M\|u\|_{H}\left\|Q S(r)^{*} u\right\|_{H} \\
& \leq M\|u\|_{H}^{2}\|Q\|_{L(H)} M \mathrm{e}^{-\alpha r} .
\end{aligned}
$$

For the third term $I_{3}$ we obtain

$$
\begin{aligned}
I_{3} & \leq C \int_{\left\{\|z\|_{H} \leq 1\right\}}\left|\left\langle S(r)^{*} u, z\right\rangle_{H}\right|^{2} \mu(d z) \\
& =C \int_{\left\{\|z\|_{H} \leq 1\right\} \cap \operatorname{ker}(P)}\left|\langle u, S(r) z\rangle_{H}\right|^{2} \mu(d z) \\
& \leq C\|u\|_{H}^{2} \mathrm{e}^{-\alpha r} \int_{\left\{\|z\|_{H} \leq 1\right\}}\|z\|_{H}^{2} \mu(d z),
\end{aligned}
$$

where $C>0$ is a generic constant. Proceeding similarly for the last term, we obtain

$$
\begin{aligned}
I_{3} & \leq C \int_{\left\{\|z\|_{H}>1\right\}} \min \left\{1,\left|\left\langle S(r)^{*} u, z\right\rangle_{H}\right|\right\} \mu(d z) \\
& \leq C \int_{\left\{\|z\|_{H}>1\right\} \cap \operatorname{ker}(P)} \min \left\{1,\|u\|_{H} \mathrm{e}^{-\alpha r}\|z\|_{H}\right\} \mu(d z) \\
& \leq C\|u\|_{H} \mathrm{e}^{-\alpha r}\left(\mu\left(\left\{\|z\|_{H}>1\right\}\right)+\int_{\left\{\|z\|_{H}>1\right\}} \log \left(1+\|z\|_{H}\right) \mu(d z)\right),
\end{aligned}
$$

where we have used, for $a=\|u\|_{H} \mathrm{e}^{-\alpha r}, b=\|z\|_{H}$, the elementary inequalities

$$
\begin{aligned}
\min \{1, a b\} & \leq C \log (1+a b) \\
& \leq C \min \{\log (1+a), \log (1+b)\}+C \log (1+a) \log (1+b) \\
& \leq C a(1+\log (1+b)),
\end{aligned}
$$


see [19, appendix]. Combining the estimates for $I_{1}, I_{2}, I_{3}, I_{4}$ we conclude that (4.2) is satisfied. Hence, using

$$
\lim _{t \rightarrow \infty}\langle S(t) x, u\rangle_{H}=\langle P x, u\rangle_{H}
$$

we find that

$$
\lim _{t \rightarrow \infty} \mathbb{E}\left[\mathrm{e}^{\mathrm{i}\left\langle u, X_{t}^{x}\right\rangle_{H}}\right]=\exp \left(\mathrm{i}\langle P x, u\rangle_{H}+\int_{0}^{\infty} \Psi\left(S(r)^{*} u\right) d r\right) .
$$

Since, in view of $(4.2), u \longmapsto \int_{0}^{\infty} \Psi\left(S(r)^{*} u\right) d r$ is continuous at $u=0$, the assertion follows from Lévy's continuity theorem combined with the particular form of (2.6).

The next remark shows that the Lévy driven OU-process is a particular case of (1.1) where $F, \sigma, \gamma$ independent of $x$.

Remark 2.2. Let $F, \sigma, \gamma$ be independent of the state space variables $x \in H$. Then (1.1) takes the form

$$
d X_{t}=\left(A X_{t}+F\right) d t+\sigma d W_{t}+\int_{E} \gamma(\nu) \widetilde{N}(d t, d z) .
$$

Setting

$$
Z_{t}=F t+\sigma W_{t}+\int_{0}^{t} \int_{E} \gamma(\nu) \tilde{N}(d s, d z),
$$

we observe that $\left(Z_{t}\right)_{t \geq 0}$ is a Lévy process with characteristic triplet $(F, \sigma, \mu \circ$ $\gamma^{-1}$ ), up to a possible change of drift related to the compensation of jumps. This shows that (2.6) is equivalent to

$$
d X_{t}=A X_{t} d t+d Z_{t}
$$

and hence the Lévy driven OU-process covers the case where $F, \sigma, \gamma$ in (1.1) are independent of the state variables.

Below we briefly discuss an application of this result to a stochastic perturbation of the Kolmogorov equation associated with a symmetric Markov semigroup. Let $E$ be a Polish space and $\eta$ a Borel probability measure on $E$. Let $(A, D(A))$ be the generator of a symmetric Markov semigroup $(S(t))_{t \geq 0}$ on $H:=L^{2}(E, \eta)$. Then there exists, for each $f \in D(A)$, a unique solution to the Kolmogorov equation (see, e.g., [31])

$$
\frac{d v(t)}{d t}=A v(t), \quad v(0)=f .
$$

Below we consider an additive stochastic perturbation of this equation in the sense of Itô, i.e. the stochastic partial differential equation

$$
d v(t)=A v(t) d t+d Z_{t}, \quad v(0)=f,
$$

where $\left(Z_{t}\right)_{t \geq 0}$ is an $L^{2}(E, \eta)$-valued Lévy process with characteristic function $\Psi$. Let $(v(t) ; f))_{t \geq 0}$ be the unique mild solution to this equation. 
Corollary 2.3. Suppose that the semigroup generated by $(A, D(A))$ on $L^{2}:=$ $L^{2}(E, \eta)$ satisfies $(2.3)$ with the projection operator

$$
P v=\int_{E} v(x) \eta(d x),
$$

and $H=L^{2}(E, \eta)$. Assume that the Lévy process $\left(Z_{t}\right)_{t \geq 0}$ satisfies the conditions (i) - (iii) of Theorem 2.1. Then

$$
v(t ; f) \longrightarrow \int_{E} f(x) \eta(d x)+v(\infty), \quad t \rightarrow \infty
$$

in law, where $v(\infty)$ is a random variable whose characteristic function is given by

$$
\mathbb{E}\left[\mathrm{e}^{\mathrm{i}\langle u, v(\infty)\rangle_{L^{2}}}\right]=\exp \left(\int_{0}^{\infty} \Psi\left(S(r)^{*} u\right) d r\right)
$$

We close this section with an example of a semigroup $(S(t))_{t \geq 0}$ for which this corollary can be applied.

Example 2.4. Let $\left(X_{t}\right)_{t \geq 0}$ be a Feller process on a separable Hilbert space $E$ and let $\left(p_{t}\right)_{t \geq 0}$ be its transition semigroup acting on $C_{b}(E)$. Suppose that $\left(X_{t}\right)_{t \geq 0}$ has a unique invariant measure $\eta$. Then, by Jensen's inequality, $\left(p_{t}\right)_{t \geq 0}$ can be uniquely extended to a strongly continuous semigroup on $L^{2}(E, \eta)$ which is for simplicity again denoted by $\left(p_{t}\right)_{t \geq 0}$. Suppose that this semigroup is $L^{2}$-exponentially convergent in the sense that

$$
\lim _{t \rightarrow \infty} \int_{E}\left(p_{t} f-\int_{E} f(x) \eta(d x)\right)^{2} d \eta=0, \quad \forall f \in L^{2}(E, \eta) .
$$

Then $\left(p_{t}\right)_{t \geq 0}$ satisfies $(2.3)$ with projection operator $P v=\int_{E} v(x) \eta(d x)$.

\section{Preliminaries}

\subsection{Framework and notation}

Here and throughout this work, $\left(\Omega, \mathcal{F},\left(\mathcal{F}_{t}\right)_{t \in \mathbb{R}_{+}}, \mathbb{P}\right)$ is a filtered probability space satisfying the usual conditions. Let $U$ be a separable Hilbert space and $W=\left(W_{t}\right)_{t \geq 0}$ be a $Q$-Wiener process with respect to $\left(\mathcal{F}_{t}\right)_{t \in \mathbb{R}_{+}}$on $(\Omega, \mathcal{F}$, $\left.\left(\mathcal{F}_{t}\right)_{t \in \mathbb{R}_{+}}, \mathbb{P}\right)$, where $Q: U \rightarrow U$ is a non-negative, symmetric, trace class operator. Let $E$ be a Polish space, $\mathcal{E}$ the Borel- $\sigma$-field on $E$, and $\mu$ a $\sigma$-finite measure on $(E, \mathcal{E})$. Let $N(d t, d \nu)$ be a $\left(\mathcal{F}_{t}\right)_{t \geq 0}$-Poisson random measure with compensator $d t \mu(d \nu)$ and denote by $\widetilde{N}(d t, d \nu)=N(d t, d \nu)-d t \mu(d \nu)$ the corresponding compensated Poisson random measure. Suppose that the random objects $\left(W_{t}\right)_{t \geq 0}$ and $N(d t, d \nu)$ are mutually independent.

In this work we investigate the long-time behavior of mild solutions to the stochastic partial differential equation (1.1) with initial condition $X_{0} \in$ $L^{2}\left(\Omega, \mathcal{F}_{0}, \mathbb{P} ; H\right)$, that is

$$
d X_{t}=\left(A X_{t}+F\left(X_{t}\right)\right) d t+\sigma\left(X_{t}\right) d W_{t}+\int_{E} \gamma\left(X_{t}^{x}, \nu\right) \tilde{N}(d t, d \nu), \quad t \geq 0
$$


where $(A, D(A))$ is the generator of a strongly continuous semigroup $(S(t))_{t \geq 0}$ on $H, H \ni x \mapsto F(x) \in H$ and $H \ni x \mapsto \sigma(x) \in L_{2}^{0}$ are Borel measurable mappings, and $(x, \nu) \mapsto \gamma(x, \nu)$ is measurable from $(H \times E, \mathcal{B}(H) \otimes \mathcal{E})$ to $(H, \mathcal{B}(H))$. Here $\mathcal{B}(H)$ denotes the Borel- $\sigma$-algebra on $H$, and $L_{2}^{0}:=L_{2}^{0}(H)$ is the Hilbert space of all Hilbert-Schmidt operators from $U_{0}$ to $H$, where $U_{0}:=Q^{1 / 2} U$ is a separable Hilbert space endowed with the scalar product

$$
\langle x, y\rangle_{U_{0}}:=\left\langle Q^{-1 / 2} x, Q^{-1 / 2} y\right\rangle_{U}=\sum_{k \in \mathbb{N}} \frac{1}{\lambda_{k}}\left\langle x, e_{k}\right\rangle_{U}\left\langle e_{k}, y\right\rangle_{U}, \quad \forall x, y \in U_{0},
$$

and $Q^{-1 / 2}$ denotes the pseudoinverse of $Q^{1 / 2}$. Here $\left(e_{j}\right)_{j \in \mathbb{N}}$ denotes an orthogonal basis of eigenvectors of $Q$ in $U$ with corresponding eigenvalues $\left(\lambda_{j}\right)_{j \in \mathbb{N}}$. For comprehensive introductions to integration concepts in infinite dimensional settings we refer e.g. to [10] for the case of $Q$-Wiener processes and e.g. to $[3,28,32]$ for compensated Poisson random measures as integrators. Throughout this work we suppose that the coefficients $F, \sigma, \gamma$ are Lipschitz continuous. More precisely:

(A1) There exist constants $L_{F}, L_{\sigma}, L_{\gamma} \geq 0$ such that for all $x, y \in H$

$$
\begin{aligned}
\|F(x)-F(y)\|_{H}^{2} & \leq L_{F}\|x-y\|_{H}^{2}, \\
\|\sigma(x)-\sigma(y)\|_{L_{2}^{0}(H)}^{2} & \leq L_{\sigma}\|x-y\|_{H}^{2}, \\
\int_{E}\|\gamma(x, \nu)-\gamma(y, \nu)\|_{H}^{2} \mu(d \nu) & \leq L_{\gamma}\|x-y\|_{H}^{2} .
\end{aligned}
$$

Moreover we suppose that

$$
\int_{E}\|\gamma(0, \nu)\|_{H}^{2} \mu(d \nu)<\infty
$$

Note that condition (3.3) implies that the jumps satisfy the usual growth conditions, i.e.

$$
\begin{aligned}
\int_{E}\|\gamma(x, \nu)\|_{H}^{2} \mu(d \nu) & \leq 2 \int_{E}\|\gamma(x, \nu)-\gamma(0, \nu)\|_{H}^{2} \mu(d \nu)+2 \int_{E}\|\gamma(0, \nu)\|_{H}^{2} \mu(d \nu) \\
& \leq 2 \max \left\{L_{\gamma}, \int_{E}\|\gamma(0, \nu)\|_{H}^{2} \mu(d \nu)\right\}\left(1+\|x\|_{H}^{2}\right) .
\end{aligned}
$$

Moreover, it follows from (GDC) and (3.2) it follows

$$
\langle A x, x\rangle_{H} \leq\left(\beta+\sqrt{L_{F}}\right)\|x\|_{H}^{2}, \quad x \in D(A) .
$$

Hence $A-\left(\beta+\sqrt{L_{F}}\right)$ is dissipative and thus by the Lumer-Phillips theorem the semigroup $(S(t))_{t \geq 0}$ generated by $(A, D(A))$ is quasi-contractive, i.e.

$$
\|S(t) x\|_{H} \leq \mathrm{e}^{\left(\beta+\sqrt{L_{F}}\right) t}\|x\|_{H}, \quad x \in H .
$$

Then, under conditions (GDC) and (A1), for each initial condition $X_{0} \in$ $L^{2}\left(\Omega, \mathcal{F}_{0}, \mathbb{P} ; H\right)$ there exists a unique cádlág, $\left(\mathcal{F}_{t}\right)_{t \geq 0}$-adapted, mean square 
continuous, mild solution $\left(X_{t}\right)_{t \geq 0}$ to (3.1) such that, for each $T>0$, there exists a constant $C(T)>0$ satisfying

$$
\mathbb{E}\left[\sup _{t \in[0, T]}\left\|X_{t}\right\|_{H}^{2}\right] \leq C(T)\left(1+\mathbb{E}\left[\left\|X_{0}\right\|_{H}^{2}\right]\right)
$$

This means that $\left(X_{t}\right)_{t \geq 0}$ satisfies $\mathbb{P}$-a.s.

$$
\begin{aligned}
X_{t}= & S(t) X_{0}+\int_{0}^{t} S(t-s) F\left(X_{s}\right) d s+\int_{0}^{t} S(t-s) \sigma\left(X_{s}\right) d W_{s} \\
& +\int_{0}^{t} \int_{E} S(t-s) \gamma\left(X_{s}, \nu\right) \tilde{N}(d s, d \nu), \quad t \geq 0
\end{aligned}
$$

where all (stochastic) integrals are well-defined, see, e.g., [1,28], and [17]. Moreover, for each $X_{0}, Y_{0} \in L^{2}\left(\Omega, \mathcal{F}_{0}, \mathbb{P} ; H\right)$, the corresponding unique solutions $\left(X_{t}\right)_{t \geq 0}$ and $\left(Y_{t}\right)_{t \geq 0}$ satisfy

$$
\mathbb{E}\left[\left\|X_{t}-Y_{t}\right\|_{H}^{2}\right] \leq C(T) \mathbb{E}\left[\left\|X_{0}-Y_{0}\right\|_{H}^{2}\right], \quad t \in[0, T] .
$$

If $X_{0} \equiv x \in H$, then we denote by $\left(X_{t}^{x}\right)_{t \geq 0}$ the corresponding solution to (3.1). Such solution constitutes a Markov process whose transition probabilities $p_{t}(x, d y)=\mathbb{P}\left[X_{t}^{x} \in d y\right]$ are measurable with respect to $x$. By slight abuse of notation we denote by $\left(p_{t}\right)_{t \geq 0}$ its transition semigroup, i.e., for each bounded measurable function $f: H \longrightarrow \mathbb{R}, p_{t} f$ is given by

$$
p_{t} f(x)=\mathbb{E}\left[f\left(X_{t}^{x}\right)\right]=\int_{H} f(y) p_{t}(x, d y), \quad t \geq 0, \quad x \in H .
$$

Using the continuous dependence on the initial condition, see (3.7), it can be shown that $p_{t} f \in C_{b}(H)$ for each $f \in C_{b}(H)$, i.e. the transition semigroup is $C_{b}$-Feller.

In this work we investigate the the existence of invariant measures and convergence of the transition probabilities towards these measures for the Markov process $\left(X_{t}^{x}\right)_{t \geq 0}$ with particular focus on the cases where uniqueness of invariant measures fails to hold. We denote by $p_{t}^{*}$ the adjoint operator to $p_{t}$ defined by

$$
p_{t}^{*} \rho(d x)=\int_{H} p_{t}(y, d x) \rho(d y), \quad t \geq 0 .
$$

Recall that a probability measure $\pi$ on $(H, \mathcal{B}(H))$ is called invariant measure for the semigroup $\left(p_{t}\right)_{t \geq 0}$ if and only if $p_{t}^{*} \pi=\pi$ holds for each $t \geq 0$. Let $\mathcal{P}_{2}(H)$ be the space of Borel probability measures $\rho$ on $(H, \mathcal{B}(H))$ with finite second moments. Recall that $\mathcal{P}_{2}(H)$ is separable and complete when equipped with the Wasserstein-2-distance

$$
\mathrm{W}_{2}(\rho, \widetilde{\rho})=\inf _{G \in \mathcal{H}(\rho, \widetilde{\rho})}\left(\int_{H \times H}\|x-y\|_{H}^{2} G(d x, d y)\right)^{\frac{1}{2}}, \quad \rho, \widetilde{\rho} \in \mathcal{P}_{2}(H) .
$$

Here $\mathcal{H}(\rho, \widetilde{\rho})$ denotes the set of all couplings of $(\rho, \widetilde{\rho})$, i.e. Borel probability measures on $H \times H$ whose marginals are given by $\rho$ and $\widetilde{\rho}$, respectively, see [40, Section 6] for a general introduction to couplings and Wasserstein distances. 


\subsection{Discussion of generalized dissipativity condition}

In this section we briefly discuss the condition

$$
\langle A x, x\rangle_{H} \leq-\lambda_{0}\|x\|_{H}^{2}+\left(\lambda_{0}+\lambda_{1}\right)\left\|P_{1} x\right\|_{H}^{2}, \quad x \in D(A),
$$

where $\lambda_{0}>0$ and $\lambda_{1} \geq 0$. Note that, if (3.9) and condition (3.1) are satisfied, then

$$
\begin{aligned}
& \langle A x-A y, x-y\rangle_{H}+\langle F(x)-F(y), x-y\rangle_{H} \\
& \leq\langle A x-A y, x-y\rangle_{H}+\sqrt{L_{F}}\|x-y\|_{H}^{2} \\
& \leq-\left(\lambda_{0}-\sqrt{L}_{F}\right)\|x-y\|_{H}^{2}+\left(\lambda_{0}+\lambda_{1}\right)\left\|P_{1} x-P_{1} y\right\|_{H}^{2},
\end{aligned}
$$

i.e. the generalized dissipativity condition (GDC) is satisfied for $\alpha=\lambda_{0}-\sqrt{L_{F}}$ and $\beta=\lambda_{1}+\sqrt{L_{F}}$, provided that $\lambda_{0}>\sqrt{L_{F}}$.

Proposition 3.1. Suppose that there exists an orthogonal decomposition $H=$ $H_{0} \oplus H_{1}$ of $H$ into closed linear subspaces $H_{0}, H_{1} \subset H$ such that $(S(t))_{t \geq 0}$ leaves $H_{0}$ and $H_{1}$ invariant and there exist constants $\lambda_{0}>0$ and $\lambda_{1} \geq 0$ satisfying

$$
\left\|S(t) x_{0}\right\|_{H} \leq \mathrm{e}^{-\lambda_{0} t}\left\|x_{0}\right\|_{H}, \quad\left\|S(t) x_{1}\right\|_{H} \leq \mathrm{e}^{\lambda_{1} t}\left\|x_{1}\right\|_{H}, \quad \forall t \geq 0 .
$$

for all $x_{0} \in H_{0}$ and $x_{1} \in H_{1}$. Then (3.9) holds for $P_{1}$ being the orthogonal projection operator onto $H_{1}$.

Proof. Let $P_{0}$ be the orthogonal projection operator onto $H_{0}$. Since $(S(t))_{t \geq 0}$ leaves the closed subspace $H_{0}$ invariant, its restriction $\left(\left.S(t)\right|_{H_{0}}\right)_{t \geq 0}$ onto $H_{0}$ is a strongly continuous semigroup of contractions on $H_{0}$ with generator $\left(A_{0}, D\left(A_{0}\right)\right)$ being the $H_{0}$ part of $A$, that is

$$
A_{0} x=A x, \quad x \in D\left(A_{0}\right)=\left\{y \in D(A) \cap H_{0} \mid A y \in H_{0}\right\} .
$$

Since $H_{0}$ is closed and $S(t)$ leaves $H_{0}$ invariant, it follows that $A y=\lim _{t \rightarrow 0}$ $\frac{S(t) y-y}{t} \in H_{0}$ for $y \in D(A) \cap H_{0}$, i.e. $D\left(A_{0}\right)=D(A) \cap H_{0}$ and $P_{0}: D(A) \rightarrow$ $D\left(A_{0}\right)$.

Arguing exactly in the same way shows that the restriction $\left(\left.S(t)\right|_{H_{1}}\right)_{t \geq 0}$ is a strongly continuous semigroup of contractions on $H_{1}$ with generator $\left(A_{1}, D\left(A_{1}\right)\right)$ given by $A_{1} x=A x$ and $x \in D\left(A_{1}\right)=D(A) \cap H_{1}$ so that $P_{1}: D(A) \rightarrow D\left(A_{1}\right)$. Since $S(t)$ leaves $H_{0}$ and $H_{1}$ invariant, we obtain $P_{0} S(t)=S(t) P_{0}, P_{1} S(t)=S(t) P_{1}$ from which we conclude that $A P_{1} x=P_{1} A x$ and $A P_{0} x=P_{0} A x$ for $x \in D(A)$.

Since $\left(\left.\mathrm{e}^{\lambda_{0} t} S(t)\right|_{H_{0}}\right)_{t \geq 0}$ is a strongly continuous semigroup of contractions on $H_{0}$ with generator $A_{0}+\lambda_{0} I$, and $\left(\left.\mathrm{e}^{-\lambda_{1} t} S(t)\right|_{H_{1}}\right)_{t \geq 0}$ is a strongly continuous semigroup of contractions on $H_{1}$ with generator $\bar{A}_{1}-\lambda_{1} I$, we have by the Lumer-Phillips theorem (see [31, Theorem 4.3])

$$
\left\langle A_{0} x_{0}, x_{0}\right\rangle_{H} \leq-\lambda_{0}\left\|x_{0}\right\|_{H}^{2} \text { and }\left\langle A_{1} x_{1}, x_{1}\right\rangle_{H} \leq \lambda_{1}\left\|x_{1}\right\|_{H}^{2}, \quad x_{0} \in H_{0}, x_{1} \in H_{1} .
$$


Hence we find that

$$
\begin{aligned}
\langle A x, x\rangle_{H} & =\left\langle A x, P_{0} x\right\rangle_{H}+\left\langle A x, P_{1} x\right\rangle_{H} \\
& =\left\langle P_{0} A x, P_{0} x\right\rangle_{H}+\left\langle P_{1} A x, P_{1} x\right\rangle_{H} \\
& =\left\langle A_{0} P_{0} x, P_{0} x\right\rangle_{H}+\left\langle A_{1} P_{1} x, P_{1} x\right\rangle_{H} \\
& \leq-\lambda_{0}\left\|P_{0} x\right\|_{H}^{2}+\lambda_{1}\left\|P_{1} x\right\|_{H}^{2} \\
& =-\lambda_{0}\|x\|_{H}^{2}+\left(\lambda_{0}+\lambda_{1}\right)\left\|P_{1} x\right\|_{H}^{2},
\end{aligned}
$$

where the last equality follows from $H_{0} \perp H_{1}$. This proves the assertion.

At this point it is worthwhile to mention that Onno van Gaans has investigated in [39] ergodicity for a class of Lévy driven stochastic partial differential equations where the semigroup $(S(t))_{t \geq 0}$ was supposed to be hyperbolic. Proposition 3.1 can be also applied for hyperbolic semigroups provided that the hyperbolic decomposition is orthogonal. The conditions of previous proposition are satisfied whenever $(S(t))_{t \geq 0}$ is a symmetric, uniformly convergent semigroup.

Remark 3.2. Suppose that $(S(t))_{t \geq 0}$ is a strongly continuous semigroup on $H$ and there exists an orthogonal projection operator $P$ on $H$ and $\lambda_{0}>0$ such that

$$
\|S(t) x-P x\|_{H} \leq \mathrm{e}^{-\lambda_{0} t}\|x-P x\|_{H}, \quad t \geq 0, \quad x \in H .
$$

Then the conditions of Proposition 3.1 are satisfied for $H_{0}=\operatorname{ker}(P)$ and $H_{1}=\operatorname{ran}(P)$ with $\lambda_{0}>0$ and $\lambda_{1}=0$. In particular, $(S(t))_{t \geq 0}$ is a semigroup of contractions.

The following example shows that (3.9) can also be satisfied for nonsymmetric and non-convergent semigroups.

Example 3.3. Let $H=\mathbb{R}^{2}, H_{0}=\mathbb{R} \times\{0\}, H_{1}=\{0\} \times \mathbb{R}$, and denote by $P_{0}, P_{1}$ the projection operators onto $H_{0}$ and $H_{1}$, respectively. Let $A$ be given by $A=\left(\begin{array}{cc}-1 & 1 \\ 0 & 1\end{array}\right)$. Then

$$
\begin{aligned}
\left\langle\left(\begin{array}{l}
x \\
y
\end{array}\right), A\left(\begin{array}{l}
x \\
y
\end{array}\right)\right\rangle_{H} & =-x^{2}+x y+y^{2} \\
& \leq-\frac{1}{2}\left(x^{2}+y^{2}\right)+2 y^{2} \\
& =-\frac{1}{2}\|(x, y)\|_{H}^{2}+2\left\|P_{1}(x, y)\right\|_{H}^{2},
\end{aligned}
$$

i.e. (3.9) holds for $\lambda_{0}=\frac{1}{2}$ and $\lambda_{1}=\frac{3}{2}$. Since $\mathrm{e}^{t A}=\left(\begin{array}{c}\mathrm{e}^{-t} \frac{\mathrm{e}^{t}-\mathrm{e}^{-t}}{2} \\ 0\end{array}\right)$, it is clear that neither the conditions of Proposition 3.1 nor of Remark 3.2 are satisfied. 


\subsection{Key stability estimate}

Define, for $x, y \in D(A)$, the function

$$
\begin{aligned}
\mathcal{L}\left(\|\cdot\|_{H}^{2}\right)(x, y):= & 2\langle A(x-y)+F(x)-F(y), x-y\rangle_{H}+\|\sigma(x)-\sigma(y)\|_{L_{2}^{0}(H)}^{2} \\
& +\int_{E}\|\gamma(x, \nu)-\gamma(y, \nu)\|_{H}^{2} \mu(d \nu) .
\end{aligned}
$$

Remark that if (1.1) has a strong solution, then the function

$$
\mathcal{L}\left(\|\cdot\|_{H}^{2}\right)(z):=2\langle A(z)+F(z), z\rangle_{H}+\|\sigma(z)\|_{L_{2}^{0}(H)}^{2}+\int_{E}\|\gamma(z, \nu)\|_{H}^{2} \mu(d \nu) .
$$

is simply the generator $\mathcal{L}$ applied to the unbounded function $\|z\|_{H}^{2}$, see, e.g, . [2, equation (3.4)]). Since we work with mild solutions instead, all computations given below require to use additionally Yosida approximations for the mild solution of (1.1).

Below we first prove a Lyapunov-type estimate for $\mathcal{L}\left(\|\cdot\|_{H}^{2}\right)$ and then deduce from that by an application of the generalized Itô-formula A.2 to (3.1) an estimate for the $L^{2}$-norm of $X_{t}^{x}-X_{t}^{y}$.

Lemma 3.4. Assume that condition (GDC) and (A1) are satisfied. Then

$$
\mathcal{L}\left(\|\cdot\|_{H}^{2}\right)(x, y) \leq-\left(2 \alpha-L_{\sigma}-L_{\gamma}\right)\|x-y\|_{H}^{2}+2(\alpha+\beta)\left\|P_{1} x-P_{1} y\right\|_{H}^{2}
$$

holds for $x, y \in D(A)$.

Proof. Using first (A1) and then (GDC) we find that

$$
\begin{aligned}
\mathcal{L}\left(\|\cdot\|_{H}^{2}\right)(x, y) \leq & \left(L_{\sigma}+L_{\gamma}\right)\|x-y\|_{H}^{2} \\
& +2\langle A x-A y, x-y\rangle_{H}+2\langle F(x)-F(y), x-y\rangle_{H} \\
\leq & -\left(2 \alpha-L_{\sigma}-L_{\gamma}\right)\|x-y\|_{H}^{2}+2(\alpha+\beta)\left\|P_{1} x-P_{1} y\right\|_{H}^{2} .
\end{aligned}
$$

This proves the asserted inequality.

The following is our key stability estimate.

Proposition 3.5. Suppose that $(G D C)$ and (A1) are satisfied, that

$$
\varepsilon:=2 \alpha-L_{\sigma}-L_{\gamma}>0,
$$

and suppose that

$$
\sup _{x \in H} \int_{E}\|\gamma(x, \nu)\|^{4} \mu(d \nu)<\infty .
$$

Then, for each $X_{0}, Y_{0} \in L^{2}\left(\Omega, \mathcal{F}_{0}, \mathbb{P} ; H\right)$ and all $t \geq 0$,

$$
\begin{aligned}
\mathbb{E} & {\left[\left\|X_{t}-Y_{t}\right\|_{H}^{2}\right] } \\
& \leq \mathrm{e}^{-\varepsilon t} \mathbb{E}\left[\left\|X_{0}-Y_{0}\right\|_{H}^{2}\right]+2(\alpha+\beta) \int_{0}^{t} \mathrm{e}^{-\varepsilon(t-s)} \mathbb{E}\left[\left\|P_{1} X_{s}-P_{1} Y_{s}\right\|_{H}^{2}\right] d s,
\end{aligned}
$$

where $\left(X_{t}\right)_{t \geq 0}$ and $\left(Y_{t}\right)_{t \geq 0}$ denote the unique solutions to (3.1), respectively. 
Proof. Let $\left(X_{t}^{n}\right)_{t \geq 0}$ and $\left(Y_{t}^{n}\right)_{t \geq 0}$ be the strong solutions to the corresponding Yosida-approximation systems

$$
\left\{\begin{array}{l}
d X_{t}^{n}=A X_{t}^{n}+R_{n} F\left(X_{t}^{n}\right) d t+R_{n} \sigma\left(X_{t}^{n}\right) d W_{t}+\int_{E} R_{n} \gamma\left(X_{t}^{n}, \nu\right) \tilde{N}(d t, d \nu), \\
X_{0}^{n}=R_{n} X_{0}, \quad t \geq 0
\end{array}\right.
$$

and

$$
\left\{\begin{array}{l}
d Y_{t}^{n}=A Y_{t}^{n}+R_{n} F\left(Y_{t}^{n}\right) d t+R_{n} \sigma\left(Y_{t}^{n}\right) d W_{t}+\int_{E} R_{n} \gamma\left(Y_{t}^{n}, \nu\right) \tilde{N}(d t, d \nu), \\
Y_{0}^{n}=R_{n} Y_{0}, \quad t \geq 0
\end{array}\right.
$$

where $R_{n}=n(n-A)^{-1}$ for $n \in \mathbb{N}$ with $n>\alpha+\beta+\sqrt{L_{F}}=: \lambda$. By (3.4) we find for each $n \geq 1+\lambda$ the inequality

$$
\left\|R_{n} z\right\|_{H} \leq \frac{n}{n-\lambda}\|z\|_{H} \leq(1+\lambda)\|z\|_{H}
$$

By classical properties of the resolvent (see [31, Lemma 3.2]), one clearly has $R_{n} z \rightarrow z$ as $n \rightarrow \infty$ in $H$. Moreover, by properties of the Yosida approximation of mild solutions of SPDEs (compare e.g. with Appendix A2 in [28] or Section 2 in [2]) we have

$$
\lim _{n \rightarrow \infty} \mathbb{E}\left[\sup _{t \in[0, T]}\left\|X_{t}^{n}-X_{t}\right\|_{H}^{2}+\sup _{t \in[0, T]}\left\|Y_{t}^{n}-Y_{t}\right\|_{H}^{2}\right]=0, \quad \forall T>0
$$

and hence there exists a subsequence (which is again denoted by $n$ ) such that $X_{t}^{n} \longrightarrow X_{t}$ and $Y_{t}^{n} \longrightarrow Y_{t}$ hold a.s. for each $t \geq 0$. Following a method proposed in [2] we verify that sufficient conditions are satisfied to apply the generalized Itô-formula from Theorem A.2 to the function $F(t, z):=\mathrm{e}^{\varepsilon t}\|z\|_{H}^{2}$, where $\varepsilon=2 \alpha-L_{\sigma}-L_{\gamma}$ is given by (3.13):

$$
\begin{aligned}
& X_{t}^{n}-Y_{t}^{n}=R_{n}\left(X_{0}-Y_{0}\right)+\int_{0}^{t}\left\{A\left(X_{s}^{n}-Y_{s}^{n}\right)+R_{n}\left(F\left(X_{s}^{n}\right)-F\left(Y_{s}^{n}\right)\right)\right\} d s \\
& \quad+\int_{0}^{t} R_{n}\left(\sigma\left(X_{s}^{n}\right)-\sigma\left(Y_{s}^{n}\right)\right) d W_{s}+\int_{0}^{t} \int_{E} R_{n}\left(\gamma\left(X_{s}^{n}, \nu\right)-\gamma\left(Y_{s}^{n}, \nu\right)\right) \tilde{N}(d s, d \nu) .
\end{aligned}
$$

Observe that, by condition (A1) and (3.14), one has

$$
\begin{aligned}
\int_{0}^{t} & \int_{E}\left\|R_{n}\left(\gamma\left(X_{s}^{n}, \nu\right)-\gamma\left(Y_{s}^{n}, \nu\right)\right)\right\|_{H}^{2} \mu(d \nu) d s \\
& +\int_{0}^{t} \int_{E}\left\|R_{n}\left(\gamma\left(X_{s}^{n}, \nu\right)-\gamma\left(Y_{s}^{n}, \nu\right)\right)\right\|_{H}^{4} \mu(d \nu) d s \\
\leq & (1+\lambda)^{2} \int_{0}^{t} \int_{E}\left\|\gamma\left(X_{s}^{n}, \nu\right)-\gamma\left(Y_{s}^{n}, \nu\right)\right\|_{H}^{2} \mu(d \nu) d s \\
& +8(1+\lambda)^{4} \int_{0}^{t} \int_{E}\left(\left\|\gamma\left(X_{s}^{n}, \nu\right)\right\|_{H}^{4}+\left\|\gamma\left(Y_{s}^{n}, \nu\right)\right\|_{H}^{4}\right) \mu(d \nu) d s \\
\leq & L_{\gamma}(1+\lambda)^{2} \int_{0}^{t}\left\|X_{s}^{n}-Y_{s}^{n}\right\|_{H}^{2} d s \\
& +16(1+\lambda)^{4} t \sup _{z \in H} \int_{E}\|\gamma(z, \nu)\|_{H}^{4} \mu(d \nu)<\infty .
\end{aligned}
$$


Thus we can apply the generalized Itô-formula from Theorem A.2 and obtain (similar to $(3.5)$ in $[2])$

$$
\begin{aligned}
\mathrm{e}^{\varepsilon t} \| & X_{t}^{n}-Y_{t}^{n}\left\|_{H}^{2}-\right\| R_{n}\left(X_{0}-Y_{0}\right) \|_{H}^{2} \\
= & \int_{0}^{t}\left\langle 2 \mathrm{e}^{\varepsilon s}\left(X_{s}^{n}-Y_{t}^{n}\right), R_{n}\left(\sigma\left(X_{s}^{n}\right)-\sigma\left(Y_{s}^{n}\right)\right) d W_{s}\right\rangle_{H} \\
& +\int_{0}^{t} \mathrm{e}^{\varepsilon s}\left[\varepsilon\left\|X_{s}^{n}-Y_{s}^{n}\right\|_{H}^{2}+\mathcal{L}_{n}\left(\|\cdot\|_{H}^{2}\right)\left(X_{s}^{n}, Y_{s}^{n}\right)\right] d s \\
& +\int_{0}^{t} \int_{E} \mathrm{e}^{\varepsilon s}\left[\left\|X_{s}^{n}-Y_{s}^{n}+R_{n}\left(\gamma\left(X_{s}^{n}, \nu\right)-\gamma\left(Y_{s}^{n}, \nu\right)\right)\right\|_{H}^{2}\right. \\
& \left.-\left\|X_{s}^{n}-Y_{s}^{n}\right\|_{H}^{2}\right] \tilde{N}(d s, d \nu),
\end{aligned}
$$

where we used, for $z, w \in D(A)$, the notation

$$
\begin{aligned}
\mathcal{L}_{n}\left(\|\cdot\|_{H}^{2}\right)(z, w):= & 2\left\langle z-w, A(z-w)+R_{n}(F(z)-F(w))\right\rangle_{H} \\
& +\left\|R_{n}(\sigma(z)-\sigma(w))\right\|_{L_{2}^{0}(H)}^{2} \\
& +\int_{E}\left\|R_{n}(\gamma(z, \nu)-\gamma(w, \nu))\right\|_{H}^{2} \mu(d \nu) .
\end{aligned}
$$

Taking expectations in (3.16) yields

$$
\begin{array}{rl}
\mathrm{e}^{\varepsilon t} & \mathbb{E}\left[\left\|X_{t}^{n}-Y_{t}^{n}\right\|_{H}^{2}\right]-\mathbb{E}\left[\left\|R_{n}\left(X_{0}-Y_{0}\right)\right\|_{H}^{2}\right] \\
& =\mathbb{E}\left[\int_{0}^{t} \mathrm{e}^{\varepsilon s}\left(\varepsilon\left\|X_{s}^{n}-Y_{s}^{n}\right\|_{H}^{2}+\mathcal{L}_{n}\left(\|\cdot\|_{H}^{2}\right)\left(X_{s}^{n}, Y_{s}^{n}\right)\right) d s\right] .
\end{array}
$$

Lemma 3.4 yields

$$
\begin{array}{rl}
\mathrm{e}^{\varepsilon t} & \mathbb{E}\left[\left\|X_{t}^{n}-Y_{t}^{n}\right\|_{H}^{2}\right]-\mathbb{E}\left[\left\|R_{n}(x-y)\right\|_{H}^{2}\right] \\
- & 2(\alpha+\beta) \int_{0}^{t} \mathrm{e}^{\varepsilon s} \mathbb{E}\left[\left\|P_{1} X_{s}^{n}-P_{1} Y_{s}^{n}\right\|_{H}^{2}\right] d s \\
& \leq \mathbb{E}\left[\int_{0}^{t} \mathrm{e}^{\varepsilon s}\left(-\mathcal{L}\left(\|\cdot\|_{H}^{2}\right)\left(X_{s}^{n}, Y_{s}^{n}\right)+\mathcal{L}_{n}\left(\|\cdot\|_{H}^{2}\right)\left(X_{s}^{n}, Y_{s}^{n}\right)\right) d s\right] .
\end{array}
$$

Below we prove that the right-hand-side tends to zero as $n \rightarrow \infty$, which would imply the assertion of this theorem. To prove the desired convergence to zero we apply the generalized Lebesgue Theorem (see [28, Theorem 7.1.8]). For this reason we have to prove that

$$
\mathcal{L}\left(\|\cdot\|_{H}^{2}\right)\left(X_{s}^{n}, Y_{s}^{n}\right)-\mathcal{L}_{n}\left(\|\cdot\|_{H}^{2}\right)\left(X_{s}^{n}, Y_{s}^{n}\right) \rightarrow 0
$$

holds a.s. for each $s>0$ as $n \rightarrow \infty$ and, moreover, there exists a constant $C>0$ such that

$$
\left|\mathcal{L}\left(\|\cdot\|_{H}^{2}\right)\left(X_{s}^{n}, Y_{s}^{n}\right)-\mathcal{L}_{n}\left(\|\cdot\|_{H}^{2}\right)\left(X_{s}^{n}, Y_{s}^{n}\right)\right| \leq C\left\|X_{s}^{n}-Y_{s}^{n}\right\|_{H}^{2} .
$$

We start with the proof of (3.18). Denote $F_{s}^{n}:=F\left(X_{s}^{n}\right)-F\left(Y_{s}^{n}\right), \sigma_{s}^{n}:=$ $\sigma\left(X_{s}^{n}\right)-\sigma\left(Y_{s}^{n}\right)$ and $\gamma_{s}^{n}(\nu):=\gamma\left(X_{s}^{n}, \nu\right)-\gamma\left(Y_{s}^{n}, \nu\right)$ and analogously $F_{s}:=$ 
$F\left(X_{s}\right)-F\left(Y_{s}\right), \sigma_{s}:=\sigma\left(X_{s}\right)-\sigma\left(Y_{s}\right)$ and $\gamma_{s}(\nu):=\gamma\left(X_{s}, \nu\right)-\gamma\left(Y_{s}, \nu\right)$ for each $n \in \mathbb{N}, s \geq 0$ and $\nu \in E$. Then

$$
\begin{aligned}
& \left|\left(\mathcal{L}\left(\|\cdot\|_{H}^{2}\right)\left(X_{s}^{n}, Y_{s}^{n}\right)-\mathcal{L}_{n}\left(\|\cdot\|_{H}^{2}\right)\left(X_{s}^{n}, Y_{s}^{n}\right)\right)\right| \\
& \quad \leq 2\left|\left\langle X_{s}^{n}-Y_{s}^{n}, F_{s}^{n}-R_{n} F_{s}^{n}\right\rangle_{H}\right|+\left|\left\|\sigma_{s}^{n}\right\|_{L_{2}^{0}}^{2}-\left\|R_{n} \sigma_{s}^{n}\right\|_{L_{2}^{0}}^{2}\right| \\
& \quad+\left|\int_{E}\left\|\gamma_{s}^{n}(\nu)\right\|_{H}^{2}-\left\|R_{n} \gamma_{s}^{n}(\nu)\right\|_{H}^{2} \mu(d \nu)\right| \\
& \quad=: I_{1}+I_{2}+I_{3} .
\end{aligned}
$$

For the first term $I_{1}$ we estimate

$$
\begin{aligned}
I_{1} & \leq 2\left\|X_{s}^{n}-Y_{s}^{n}\right\|_{H}\left\|F_{s}^{n}-R_{n} F_{s}^{n}\right\|_{H} \\
& \leq 2\left\|X_{s}^{n}-Y_{s}^{n}\right\|_{H}\left(\left\|F_{s}^{n}-F_{s}\right\|_{H}+\left\|F_{s}-R_{n} F_{s}\right\|_{H}+\left\|R_{n} F_{s}-R_{n} F_{s}^{n}\right\|_{H}\right) \\
& \leq 2\left\|X_{s}^{n}-Y_{s}^{n}\right\|_{H}\left(\left\|F_{s}^{n}-F_{s}\right\|_{H}+\left\|F_{s}-R_{n} F_{s}\right\|_{H}+(1+\lambda)\left\|F_{s}-F_{s}^{n}\right\|_{H}\right) .
\end{aligned}
$$

Using that $X_{s}^{n} \rightarrow X_{s}$ and $Y_{s}^{n} \rightarrow Y_{s}$ as a.s. for some subsequence (also denoted by $n$ ), we easily find that the right-hand side tends to zero. The convergence of the second term follows from

$$
\begin{aligned}
I_{2} & =\left|\left\|\sigma_{s}^{n}\right\|_{L_{2}^{0}}-\left\|R_{n} \sigma_{s}^{n}\right\|_{L_{2}^{0}}\right|\left(\left\|\sigma_{s}^{n}\right\|_{L_{2}^{0}}+\left\|R_{n} \sigma_{s}^{n}\right\|_{L_{2}^{0}}\right) \\
& \leq(2+\lambda) \sqrt{L_{\sigma}}\left\|\sigma_{s}^{n}-R_{n} \sigma_{s}^{n}\right\|_{L_{2}^{0}}\left\|X_{s}^{n}-Y_{s}^{n}\right\|_{H} \\
& \leq(2+\lambda)^{2} \sqrt{L_{\sigma}}\left\|X_{s}^{n}-Y_{s}^{n}\right\|_{H}\left(\left\|\sigma_{s}^{n}-\sigma_{s}\right\|_{L_{2}^{0}}+\left\|\sigma_{s}-R_{n} \sigma_{s}\right\|_{L_{2}^{0}}+\left\|\sigma_{s}-\sigma_{s}^{n}\right\|_{L_{2}^{0}}\right) .
\end{aligned}
$$

It remains to show the convergence of the third term. First, observe

$$
\begin{aligned}
I_{3} \leq & (2+\lambda) \int_{E}\left\|\gamma_{s}^{n}(\nu)-R_{n} \gamma_{s}^{n}(\nu)\right\|_{H}\left\|\gamma_{s}^{n}(\nu)\right\|_{H} \mu(d \nu) \\
\leq & (2+\lambda) \int_{E}\left(\left\|\gamma_{s}^{n}(\nu)-\gamma_{s}(\nu)\right\|_{H}+\left\|\gamma_{s}(\nu)-R_{n} \gamma_{s}(\nu)\right\|_{H}\right. \\
& \left.+\left\|R_{n} \gamma_{s}(\nu)-R_{n} \gamma_{s}^{n}(\nu)\right\|_{H}\right)\left\|\gamma_{s}^{n}(\nu)\right\|_{H} \mu(d \nu) \\
\leq & (2+\lambda)\left(\int_{E}\left\|\gamma_{s}^{n}(\nu)\right\|_{H}^{2} \mu(d \nu)\right)^{\frac{1}{2}}\left[\left(\int_{E}\left\|\gamma_{s}^{n}(\nu)-\gamma_{s}(\nu)\right\|_{H}^{2} \mu(d \nu)\right)^{\frac{1}{2}}\right. \\
& \left.+\left(\int_{E}\left\|\gamma_{s}(\nu)-R_{n} \gamma_{s}(\nu)\right\|_{H}^{2} \mu(d \nu)\right)^{\frac{1}{2}}+\left(\int_{E}\left\|R_{n} \gamma_{s}(\nu)-R_{n} \gamma_{s}^{n}(\nu)\right\|_{H}^{2} \mu(d \nu)\right)^{\frac{1}{2}}\right] \\
\leq & \sqrt{2}(2+\lambda)^{2} L_{\gamma}\left\|X_{s}^{n}-Y_{s}^{n}\right\|_{H}\left(\left\|X_{s}^{n}-X_{s}\right\|_{H}+\left\|Y_{s}^{n}-Y_{s}\right\|_{H}\right) \\
& +(2+\lambda) \sqrt{L_{\gamma}}\left\|X_{s}^{n}-Y_{s}^{n}\right\|_{H}\left(\int_{E}\left\|\gamma_{s}(\nu)-R_{n} \gamma_{s}(\nu)\right\|_{H}^{2} \mu(d \nu)\right)^{\frac{1}{2}} \\
= & I_{3}^{1}+I_{3}^{2}
\end{aligned}
$$


where the last inequality follows from condition (A1) combined with the inequality

$$
\begin{aligned}
& \left\|R_{n} \gamma_{s}(\nu)-R_{n} \gamma_{s}^{n}(\nu)\right\|_{H}^{2} \\
& \quad \leq(1+\lambda)^{2}\left\|\gamma_{s}(\nu)-\gamma_{s}^{n}(\nu)\right\|_{H}^{2} \\
& \quad \leq 2(1+\lambda)^{2}\left(\left\|\gamma\left(X_{s}, \nu\right)-\gamma\left(Y_{s}, \nu\right)\right\|_{H}^{2}+\left\|\gamma\left(X_{s}^{n}, \nu\right)-\gamma\left(Y_{s}^{n}, \nu\right)\right\|_{H}^{2}\right) .
\end{aligned}
$$

The first expression $I_{1}^{1}$ clearly tends to zero as $n \rightarrow \infty$. For the second expression $I_{3}^{2}$ we use the inequality $\left\|\gamma_{s}(\nu)-R_{n} \gamma_{s}(\nu)\right\|_{H}^{2} \leq 2(2+\lambda)^{2}\left\|\gamma_{s}(\nu)\right\|_{H}^{2}$ so that dominated convergence theorem is applicable, which shows that $I_{3}^{2} \rightarrow 0$ as $n \rightarrow \infty$ a.s.. This proves (3.18). Concerning (3.19), we find that

$$
\begin{aligned}
\left|\left(\mathcal{L}\left(\|\cdot\|_{H}^{2}\right)\left(X_{s}^{n}, Y_{s}^{n}\right)-\mathcal{L}_{n}\left(\|\cdot\|_{H}^{2}\right)\left(X_{s}^{n}, Y_{s}^{n}\right)\right)\right| \\
\leq 2\left|\left\langle X_{s}^{n}-Y_{s}^{n}, F_{s}^{n}-R_{n} F_{s}^{n}\right\rangle_{H}\right|+\left|\left\|\sigma_{s}^{n}\right\|_{L_{2}^{0}(H)}^{2}-\left\|R_{n} \sigma_{s}^{n}\right\|_{L_{2}^{0}(H)}^{2}\right| \\
\quad+\left|\int_{E}\left\|\gamma_{s}^{n}(\nu)\right\|_{H}^{2}-\left\|R_{n} \gamma_{s}^{n}(\nu)\right\|_{H}^{2} \mu(d \nu)\right| \\
\leq 2(2+\lambda)\left\|X_{s}^{n}-Y_{s}^{n}\right\|_{H}\left\|F_{s}^{n}\right\|_{H}+\left(1+(1+\lambda)^{2}\right) \\
\quad\left[\left\|\sigma_{s}^{n}\right\|_{L_{2}^{0}(H)}^{2}+\int_{E}\left\|\gamma_{s}^{n}(\nu)\right\|_{H}^{2} \mu(d \nu)\right] \\
\leq 2(2+\lambda) L_{F}\left\|X_{s}^{n}-Y_{s}^{n}\right\|_{H}^{2}+\left(1+(1+\lambda)^{2}\right)\left(L_{\sigma}+L_{\gamma}\right)\left\|X_{s}^{n}-Y_{s}^{n}\right\|_{H}^{2} .
\end{aligned}
$$

Hence the generalized Lebesgue Theorem is applicable, and thus the assertion of this theorem is proved.

Note that condition (3.14) is used to guarantee that the Itô-formula A.2 for Hilbert space valued jump diffusions can be applied for $(x, t) \rightarrow \mathrm{e}^{t \varepsilon}\|x\|_{H}^{2}$. The assertion of Proposition 3.5 is also true when $\varepsilon \leq 0$, but will be only applied for the case when $\varepsilon>0$.

\section{Convergence to limiting distribution}

\subsection{The strongly dissipative case}

As a consequence of our key stability estimate we can provide a simple proof for the existence and uniqueness of a unique limiting distribution in the spirit of classical results such as [32, Section 16], [10, Chapter 11, Section 5], and $[36]$.

Theorem 4.1. Assume that condition $(G D C)$ is satisfied for $P_{1}=0$ (and hence $\beta=0)$, (A1) holds, and (3.14) is satisfied. If (3.13) is satisfied, then

$$
\mathrm{W}_{2}\left(p_{t}^{*} \rho, p_{t}^{*} \widetilde{\rho}\right) \leq \mathrm{W}_{2}(\rho, \widetilde{\rho}) \mathrm{e}^{-\varepsilon t / 2}, \quad t \geq 0,
$$

holds for any $\rho, \widetilde{\rho} \in \mathcal{P}_{2}(H)$. In particular, the Markov process determined by (3.1) has a unique invariant measure $\pi$. This measure has finite second moments and it holds that

$$
\mathrm{W}_{2}\left(p_{t}^{*} \rho, \pi\right) \leq \mathrm{W}_{2}(\rho, \pi) \mathrm{e}^{-\varepsilon t / 2}, \quad t \geq 0,
$$

for each $\rho \in \mathcal{P}_{2}(H)$. 
Proof. Using (GDC) with $P_{1}=0$ combined with Proposition 3.5 we find that

$$
\mathbb{E}\left[\left\|X_{t}^{x}-X_{t}^{y}\right\|_{H}^{2}\right] \leq \mathrm{e}^{-\varepsilon t}\|x-y\|_{H}^{2}, \quad x, y \in H .
$$

Using the definition of the Wasserstein distance, we conclude that

$$
\mathrm{W}_{2}\left(p_{t}^{*} \delta_{x}, p_{t}^{*} \delta_{y}\right) \leq\left(\mathbb{E}\left[\left\|X_{t}^{x}-X_{t}^{y}\right\|_{H}^{2}\right]\right)^{1 / 2} \leq\|x-y\|_{H} \mathrm{e}^{-\varepsilon t / 2} .
$$

The latter one readily yields (4.12). Finally, the existence and uniqueness of an invariant measure as well as (4.2) can be derived from (4.12) combined with a standard Cauchy argument.

This result can be seen as an analogue of the conditions introduced in $[32$, Section 16], [10, Chapter 11, Section 5], and [36], where a similar statement was given. Opposite to this case, in this work we focus on the study of multiple invariant measures. For this purpose we will assume that $\varepsilon>0$ and that (GDC) holds for some $P_{1} \neq 0$.

\subsection{The case of vanishing coefficients}

While Proposition 3.5 provides an estimate on the $L^{2}$-norm of the difference $X_{t}^{x}-X_{t}^{y}$, such an estimate alone does neither imply the existence nor uniqueness of an invariant distribution. However, if the coefficients $F, \sigma, \gamma$ vanish at $H_{1}$, then we may characterize the limiting distributions in $L^{2}$.

Theorem 4.2. Suppose that $(G D C)$ holds with a projection operator $P_{1}$, (A1), (3.14), (3.13) are satisfied, that $(S(t))_{t \geq 0}$ leaves $H_{0}:=\operatorname{ran}\left(I-P_{1}\right)$ invariant, and that $\operatorname{ran}\left(P_{1}\right) \subset \operatorname{ker}(A)$. Moreover, assume that

$$
P_{1} F \equiv 0, P_{1} \sigma \equiv 0, P_{1} \gamma \equiv 0 .
$$

Given any $X_{0} \in L^{2}\left(\Omega, \mathcal{F}_{0}, \mathbb{P} ; H\right)$ which satisfies

$$
F\left(P_{1} X_{0}\right)=0, \sigma\left(P_{1} X_{0}\right)=0, \gamma\left(P_{1} X_{0}, \cdot\right)=0, \quad \text { a.s. }
$$

then the inequality

$$
\mathbb{E}\left[\left\|X_{t}-P_{1} X_{0}\right\|_{H}^{2}\right] \leq \mathrm{e}^{-\varepsilon t} \mathbb{E}\left[\left\|\left(I-P_{1}\right) X_{0}\right\|_{H}^{2}\right]
$$

holds. In particular, let $\rho$ be the law of $X_{0} \in L^{2}\left(\Omega, \mathcal{F}_{0}, \mathbb{P} ; H\right)$ and $\rho_{1}$ be the law of $P_{1} X_{0}$, respectively. Then $\rho_{1}$ is an invariant measure.

Proof. Fix $X_{0} \in L^{2}\left(\Omega, \mathcal{F}_{0}, \mathbb{P} ; H\right)$ with property (4.4) and set $P_{0}=I-P_{1}$. Since $\operatorname{ran}\left(P_{1}\right) \subset \operatorname{ker}(A)$ we find that $S(t) P_{1}=P_{1}$ for $t \geq 0$ and hence $P_{0} S(t) P_{1}=0$. Moreover, since $(S(t))_{t \geq 0}$ leaves $H_{0}$ invariant, we obtain $P_{0} S(t)=P_{0} S(t) P_{0}+$ $P_{0} S(t) P_{1}=P_{0} S(t) P_{0}=S(t) P_{0}$. Hence, using (4.3) we find that

$$
P_{1} X_{t}=P_{1} S(t) X_{0}=P_{1} S(t) P_{0} X_{0}+P_{1} S(t) P_{1} X_{0}=P_{1} X_{0} .
$$


From this we conclude that $\left(P_{0} X_{t}\right)_{t \geq 0}$ satisfies

$$
\begin{aligned}
P_{0} X_{t}= & P_{0} S(t) X_{0}+\int_{0}^{t} P_{0} S(t-s) F\left(X_{s}\right) d s+\int_{0}^{t} P_{0} S(t-s) \sigma\left(X_{s}\right) d W_{s} \\
& +\int_{0}^{t} \int_{E} P_{0} S(t-s) \gamma\left(X_{s}\right) \tilde{N}(d s, d \nu) \\
= & S(t) P_{0} X_{0}+\int_{0}^{t} S(t-s) P_{0} F\left(P_{1} X_{0}+P_{0} X_{t}\right) d s \\
& +\int_{0}^{t} S(t-s) P_{0} \sigma\left(P_{1} X_{0}+P_{0} X_{s}\right) d W_{s} \\
& +\int_{0}^{t} \int_{E} S(t-s) P_{0} \gamma\left(P_{1} X_{0}+P_{0} X_{s}\right) \tilde{N}(d s, d \nu) \\
= & S(t) P_{0} X_{0}+\int_{0}^{t} S(t-s) \widetilde{F}\left(P_{0} X_{t}\right) d s+\int_{0}^{t} S(t-s) \widetilde{\sigma}\left(P_{0} X_{s}\right) d W_{s} \\
& \int_{0}^{t} \int_{E} S(t-s) \widetilde{\gamma}\left(P_{0} X_{s}\right) \tilde{N}(d s, d \nu),
\end{aligned}
$$

where we have set $\widetilde{F}(y):=P_{0} F\left(P_{1} X_{0}+y\right), \widetilde{\sigma}(y):=P_{0} \sigma\left(P_{1} X_{0}+y\right)$ and $\widetilde{\gamma}(y, \nu):=P_{0} \gamma\left(P_{1} X_{0}+y, \nu\right)$ for all $y \in H_{0}$ and $\nu \in E$. Since these coefficients share the same Lipschitz estimates as $F, \sigma$ and $\gamma$, are $\mathcal{F}_{0}$-measurable and the noise terms are independent of $\mathcal{F}_{0}$, we can apply Proposition 3.5 (conditionally on $\left.\mathcal{F}_{0}\right)$ to the process $\left(P_{0} X_{t}\right)_{t \geq 0}$ obtained from the above auxiliary SPDE and obtain

$$
\mathbb{E}\left[\left\|X_{t}-P_{1} X_{0}\right\|_{H}^{2}\right]=\mathbb{E}\left[\left\|P_{0} X_{t}\right\|_{H}^{2}\right]=\mathbb{E}\left[\left\|P_{0} X_{t}-P_{0} Y_{t}^{0}\right\|_{H}^{2}\right] \leq \mathrm{e}^{-\varepsilon t} \mathbb{E}\left[\left\|P_{0} X_{0}\right\|_{H}^{2}\right],
$$

where we have used that $P_{0} Y_{t}=0$ for the unique solution with $Y_{0}=0$ due to (4.4).

This theorem can be applied, for instance, to the Heath-Jarrow-MortonMusiela equation, see Sect. 5.

\subsection{Main result: the general case}

In Theorem 4.2 we have assumed (4.3), (4.4), and that $(S(t))_{t>0}$ leaves $H_{0}$ invariant. Below we continue with the more general case. Namely, for the projection operator $P_{1}$ given by condition (GDC) we set $P_{0}=I-P_{1}$ and suppose that:

(A2) The semigroup $(S(t))_{t \geq 0}$ leaves $H_{1}:=\operatorname{ran}\left(I-P_{0}\right)$ invariant, one has

$$
P_{1} \sigma=P_{1} \gamma=0 \text { and } P_{1} F(x)=P_{1} F\left(P_{1} x\right), \quad x \in H .
$$

Let us briefly comment on this condition. Let $\left(X_{t}\right)_{t \geq 0}$ be the unique solution to (3.6) and decompose the process $X_{t}$ according to $X_{t}=P_{0} X_{t}+P_{1} X_{t}$. Then condition (A2) simply implies that $P_{1} X_{t}$ is $\mathcal{F}_{0}$-measurable and satisfies $\omega$-wisely the deterministic equation

$$
f(t ; x)=P_{1} S(t) x+\int_{0}^{t} P_{1} S(t-s) P_{1} F\left(P_{1} f(s ; x)\right) d s, \quad f(0, x)=x \in H,
$$


i.e. $P_{1} X_{t}=f(t ; x)$ with $f(0, x)=x=X_{0}$ holds a.s. Our next condition imposes a control on this component:

(A3) For each $x \in H_{1}=\operatorname{ran}\left(P_{1}\right)$ there exists $\tilde{f}(x) \in H_{1}$ and constants $C(x)>$ $0, \delta(x)>0$ such that

$$
\|f(t ; x)-\tilde{f}(x)\|_{H}^{2} \leq C(x) \mathrm{e}^{-\delta(x) t}, \quad t \geq 0 .
$$

Without loss of generality we will always suppose that $\delta(x) \in(0,|\varepsilon|)$. Such assumption will simplify our arguments later on. Note that, if $P_{1} F\left(P_{1} \cdot\right)=0$ then condition (A3) reduces to a condition on the limiting behavior of the semigroup $(S(t))_{t \geq 0}$ when restricted to $H_{1}=\operatorname{ran}\left(P_{1}\right)$. In such a case condition (A3) is, for instance, satisfied if $\operatorname{ran}\left(P_{1}\right) \subset \operatorname{ker}(A)$. Recall that condition (GDC) was formulated in the introduction and that (A1), (3.14) and (3.13) were formulated in Sect. 3. The following is our main result in this section.

Theorem 4.3. Suppose that condition $(G D C)$ holds for some projection operator $P_{1}$, that conditions (A1) - (A3), (3.14) and (3.13) are satisfied. Then the following assertions hold:

(a) For each $x \in H$ there exists an invariant measure $\pi_{\delta_{x}} \in \mathcal{P}_{2}(H)$ for the Markov semigroup $\left(p_{t}\right)_{t \geq 0}$ and a constant $K(\alpha, \beta, \varepsilon, h)>0$ such that

$$
\mathrm{W}_{2}\left(p_{t}(x, \cdot), \pi_{\delta_{x}}\right) \leq K(\alpha, \beta, \varepsilon, x) \mathrm{e}^{-\frac{\delta(x)}{2} t}, \quad t \geq 0 .
$$

(b) Suppose, in addition to the conditions of (A3), that there are constants $\delta$ and $C$, such that

$$
\delta(x) \geq \delta>0 \quad \text { and } \quad C(x) \leq C\left(1+\|x\|_{H}\right)^{4}, \quad x \in H .
$$

Then, for each $\rho \in \mathcal{P}_{2}(H)$, there exists an invariant measure $\pi_{\rho} \in \mathcal{P}_{2}(H)$ for the Markov semigroup $\left(p_{t}\right)_{t \geq 0}$ and a constant $K(\alpha, \beta, \varepsilon)>0$ such that

$$
\mathrm{W}_{2}\left(p_{t}^{*} \rho, \pi_{\rho}\right) \leq K(\alpha, \beta, \varepsilon) \int_{H}\left(1+\|x\|_{H}\right)^{2} \rho(d x) \mathrm{e}^{-\frac{\delta}{2} t}, \quad t \geq 0 .
$$

The proof of this theorem relies on the key stability estimate formulated in Proposition 3.5 and is given at the end of this section. So far we have stated the existence of invariant measures parametrized by the initial state of the process. However, under the given conditions it can also be shown that $\pi_{\delta_{x}}$ as well as $\pi_{\rho}$ depend only on the $H_{1}$ part of $x$ or $\rho$, respectively.

Corollary 4.4. Suppose that condition (GDC) holds for some projection operator $P_{1}$, that conditions (A1) - (A3), (3.14) and (3.13) are satisfied. Then the following assertions hold:

(a) Let $x, y \in H$ be such that $P_{1} x=P_{1} y$. Then $\pi_{\delta_{x}}=\pi_{\delta_{y}}$.

(b) Suppose, in addition, that (4.6) holds. Let $\rho, \widetilde{\rho} \in \mathcal{P}_{2}(H)$ be such that $\rho \circ P_{1}^{-1}=\widetilde{\rho} \circ P_{1}^{-1}$. Then $\pi_{\rho}=\pi_{\widetilde{\rho}}$.

Let us briefly compare the conditions imposed in Theorem 4.2 with those imposed in Theorem 4.3. In Theorem 4.3 we have weakened (4.3) with respect to $F$ by replacing $P_{1} F=0$ by $P_{1} F(x)=P_{1} F\left(P_{1} x\right)$. Moreover, we have replaced $\operatorname{ran}\left(P_{1}\right) \subset \operatorname{ker}(A)$ by condition (A3). Finally note that condition (4.4) 
is not assumed in Theorem 4.3. Below we provide a counter example showing that, in general, condition (A3) cannot be omitted.

Example 4.5. Let $H=\mathbb{R}^{2}$ and $\left(W_{t}\right)_{t \geq 0}$ be a 2-dimensional standard Brownian motion. Let $Y_{t}=\left(Y_{t}^{1}, Y_{t}^{2}\right) \in H=\mathbb{R}^{2}$ be the solution of

$$
d Y_{t}=\left(\begin{array}{cc}
-1 & 1 \\
0 & 1
\end{array}\right) Y_{t} d t+\left(\begin{array}{ll}
1 & 0 \\
0 & 0
\end{array}\right) d W_{t}
$$

Then condition (A1) holds for $F=0, \gamma=0$ and clearly $\sigma(x)=\left(\begin{array}{ll}1 & 0 \\ 0 & 0\end{array}\right)$. Example 3.3 shows that (GDC) holds with $P_{1}$ being the projection onto the second coordinate. Moreover, (4.3) and hence (A2) holds. However, since

$$
Y_{t}^{2}=\mathrm{e}^{t} Y_{0}^{2}+\int_{0}^{t} \mathrm{e}^{t-s} d W_{s}^{2}
$$

it is clear that condition (A3) is not satisfied. Moreover, $Y_{t}^{2}$ does not have a limiting distribution and hence also $Y_{t}$ cannot have a limiting distribution.

The next remark shows that, under a stronger condition on the Lévy measure, the results obtained in Theorem 2.1 could partially also be deduced from the general statements of this section.

Remark 4.6. The results obtained in Sect. 2 for the Lévy driven OrnsteinUhlenbeck process could partially be also obtained from the above results. Indeed, (2.1) can be cast into the form

$$
\begin{aligned}
d X_{t}^{x} & =A X_{t}^{x} d t+d Z_{t} \\
& =\left(A X_{t}^{x}+b\right) d t+Q d W_{t}+\int_{\left\{\|z\|_{H} \leq 1\right\} \backslash\{0\}} z \widetilde{N}(d t, d z)+\int_{\left\{\|z\|_{H}>1\right\}} z N(d t, d z) \\
& =\left(A X_{t}^{x}+b+c\right) d t+Q d W_{t}+\int_{H \backslash\{0\}} z \widetilde{N}(d t, d z),
\end{aligned}
$$

where $c=\int_{\left\{\|z\|_{H}>1\right\}} z \mu(d z)$ and we have used the Lévy-Ito decomposition for the Lévy process $\left(Z_{t}\right)_{t \geq 0}$, i.e.,

$$
Z_{t}=b t+Q W_{t}+\int_{\left\{\|z\|_{H} \leq 1\right\} \backslash\{0\}} z \widetilde{N}(d t, d z)+\int_{\left\{\|z\|_{H}>1\right\}} z N(d t, d z),
$$

where $N(d t, d z)$ is a Poisson random measure with compensator $d t \mu(d z)$. Suppose that the conditions of Theorem 2.1 are satisfied. If the semigroup generated by $(A, D(A))$ is also symmetric, then using Proposition 3.1 one can show that also (GDC) holds. Condition (A1) is clearly satisfied for $L_{\gamma}=L_{\sigma}=0$ and $\sigma(x)=Q, \gamma(x, z)=z$. Thus (3.13) is satisfied. To prove condition (A2) we let $H_{0}=\operatorname{ker}(P), H_{1}=\operatorname{ran}(P)$ and observe that $P X_{t}$, compare with (4.5), 
simplifies to

$$
\begin{aligned}
P X_{t}= & P S(t) x+\int_{0}^{t} P S(t-s)(b+c) d s+\int_{0}^{t} P S(t-s) Q d W_{s} \\
& +\int_{0}^{t} \int_{H \backslash\{0\}} P S(t-s) z \tilde{N}(d s, d z) \\
= & P x+\int_{0}^{t} \int_{H \backslash\{0\}} P z \tilde{N}(d s, d z),
\end{aligned}
$$

where we have used $P S(t-s)=P S(t-s) P=P$ and the conditions imposed in Theorem 2.1 on the Lev́y triplet, i.e., $P(b+c)=0$ and $P Q=0$. Noting that $\operatorname{supp}(\mu) \subset \operatorname{ker}(P)$ we find that $\widetilde{N}$ is supported on $\mathbb{R}_{+} \times \operatorname{ker}(P)$ and hence

$$
\int_{0}^{t} \int_{H \backslash\{0\}} P z \widetilde{N}(d s, d z)=0 .
$$

This shows that condition (A3) is satisfied for any choice of $C(x), \delta(x)$ and $\tilde{f}(x)=P x$. Finally, (3.14) requires that $\mu$ satisfies the stronger moment condition

$$
\int_{\left\{\|z\|_{H}>1\right\}}\|z\|_{H}^{4} \mu(d z)<\infty .
$$

Thus under the above assumptions the existence of multiple invariant measures for the Ornstein-Uhlenbeck process also follows from Theorem 4.3 and Corollary 4.4. However, in contrast to Theorem 2.1, the general results from this section, do not provide an explicit characterization of the limiting distributions in terms of the Fourier transform and also require to assume stronger conditions.

Next we turn to a proof of Theorem 4.3 and Corollary 4.4.

\subsection{Construction of a coupling}

Let $x \in H$ and let $\left(X_{t}^{x}\right)_{t \geq 0}$ be the unique mild solution to (3.6). Below we construct for given $\tau \geq 0$ a coupling for the law of $\left(X_{t}^{x}, X_{t+\tau}^{x}\right)$. Let $\left(Y_{t}^{x, \tau}\right)_{t \geq 0}$ be the unique mild solution to the SPDE

$$
\begin{aligned}
Y_{t}^{x, \tau}= & S(t) x+\int_{0}^{t} S(t-s) F\left(Y_{s}^{x, \tau}\right) d s+\int_{0}^{t} S(t-s) \sigma\left(Y_{s}^{x, \tau}\right) d W_{s}^{\tau} \\
& +\int_{0}^{t} \int_{E} S(t-s) \gamma\left(Y_{s}^{x, \tau}, \nu\right) \tilde{N}^{\tau}(d s, d \nu), \quad t \geq 0,
\end{aligned}
$$

where $W_{s}^{\tau}=W_{\tau+s}-W_{\tau}$ is a $Q$-Wiener process, and $\tilde{N}^{\tau}(d s, d \nu)$ defined by

$$
\tilde{N}^{\tau}((0, t] \times A):=\tilde{N}((\tau, \tau+t] \times A)
$$

for $t>0$ and $A \in \mathcal{E}$ is a Poisson random measure with respect to the filtration $\left(\mathcal{F}_{s}^{\tau}\right)_{s \geq 0}$ defined by $\mathcal{F}_{s}^{\tau}=\mathcal{F}_{s+\tau}$.

Lemma 4.7. Suppose that (GDC), (A1), (3.14) and (3.13) are satisfied. Then for each $x \in H$ and $t, \tau \geq 0$ the following assertions hold:

(a) $Y_{t}^{x, \tau}$ has the same law as $X_{t}^{x}$. 
(b) It holds that

$$
\begin{aligned}
\mathbb{E}\left[\left\|Y_{t}^{x, \tau}-X_{t+\tau}^{x}\right\|_{H}^{2}\right] \leq & \mathrm{e}^{-\varepsilon t} \mathbb{E}\left[\left\|x-X_{\tau}^{x}\right\|_{H}^{2}\right] \\
& +2(\alpha+\beta) \int_{0}^{t} \mathrm{e}^{-\varepsilon(t-s)} \mathbb{E}\left[\left\|P_{1} Y_{s}^{x, \tau}-P_{1} X_{s+\tau}^{x}\right\|_{H}^{2}\right] d s .
\end{aligned}
$$

Proof. (a) Since (3.6) has a unique solution it follows from the YamadaWatanabe Theorem (see [26]) that also uniqueness in law holds for this equation. Since the driving noises $N^{\tau}$ and $W^{\tau}$ in (4.7) have the same law as $N$ and $W$ from (3.6), it follows that the unique solution to (4.7) has the same law as the solution to (3.6). This proves the assertion.

(b) Set $X_{t}^{x, \tau}:=X_{t+\tau}^{x}$, then by direct computation we find that

$$
\begin{aligned}
X_{t}^{x, \tau}= & S(t) S(\tau) x+\int_{0}^{t+\tau} S(t+\tau-s) F\left(X_{s}^{x}\right) d s+\int_{0}^{t+\tau} S(t+\tau-s) \sigma\left(X_{s}^{x}\right) d W_{s} \\
& +\int_{0}^{t+\tau} \int_{E} S(t+\tau-s) \gamma\left(X_{s}^{x}, \nu\right) \tilde{N}(d s, d \nu) \\
= & S(t) S(\tau) x+S(t) \int_{0}^{\tau} S(\tau-s) F\left(X_{s}^{x}\right) d s+S(t) \int_{0}^{\tau} S(\tau-s) \sigma\left(X_{s}^{x}\right) d W_{s} \\
& +S(t) \int_{0}^{\tau} \int_{E} S(\tau-s) \gamma\left(X_{s}^{x}, \nu\right) \tilde{N}(d s, d \nu) \\
& +\int_{\tau}^{t+\tau} S(t+\tau-s) F\left(X_{s}^{x}\right) d s+\int_{\tau}^{t+\tau} S(t+\tau-s) \sigma\left(X_{s}^{x}\right) d W_{s} \\
& +\int_{\tau}^{t+\tau} \int_{E} S(t+\tau-s) \gamma\left(X_{s}^{x}, \nu\right) \tilde{N}(d s, d \nu) \\
= & S(t) X_{0}^{x, \tau}+\int_{0}^{t} S(t-s) F\left(X_{s}^{x, \tau}\right) d s+\int_{0}^{t} S(t-s) \sigma\left(X_{s}^{x, \tau}\right) d W_{s}^{\tau} \\
& +\int_{0}^{t} \int_{E} S(t-s) \gamma\left(X_{s}^{x, \tau}, \nu\right) \tilde{N}^{\tau}(d s, d \nu),
\end{aligned}
$$

where in the last equality we have used, for appropriate integrands $\Phi(s, \nu)$ and $\Psi(s)$, that

$$
\begin{gathered}
\int_{\tau}^{\tau+t} \Psi(s) d W_{s}=\int_{0}^{t} \Psi(s+\tau) d W_{s}^{\tau}, \\
\int_{\tau}^{\tau+t} \int_{E} \Phi(s, \nu) \tilde{N}(d s, d \nu)=\int_{0}^{t} \int_{E} \Phi(s+\tau, \nu) \tilde{N}^{\tau}(d s, d \nu) .
\end{gathered}
$$

Hence $\left(X_{t}^{x, \tau}\right)_{t \geq 0}$ also solves $(4.7)$ with $\mathcal{F}_{0}^{\tau}=\mathcal{F}_{\tau}$ and initial condition $X_{0}^{x, \tau}=$ $X_{\tau}^{x}$. Consequently, the assertion follows from Proposition 3.5 applied to $X_{t}^{x, \tau}$ and $Y_{t}^{x, \tau}$.

\subsection{Proof of Theorem 4.3}

Proof of Theorem 4.3. Fix $x \in H$ and recall that $p_{t}(x, \cdot)$ denotes the transition probabilities of the Markov process obtained from (3.6). Below we prove that $\left(p_{t}(x, \cdot)\right)_{t \geq 0} \subset \mathcal{P}_{2}(H)$ is a Cauchy sequence with respect to the Wasserstein distance $\overline{\mathrm{W}}_{2}$. Fix $t, \tau \geq 0$. We treat the cases $\tau \in(0,1]$ and $\tau>1$ separately. 
Case $0<\tau \leq 1$ : Then using the coupling lemma 4.7.(b) yields

$$
\begin{aligned}
& \mathrm{W}_{2}\left(p_{t+\tau}(x, \cdot), p_{t}(x, \cdot)\right) \leq\left(\mathbb{E}\left[\left\|Y_{t}^{x, \tau}-X_{t+\tau}^{x}\right\|_{H}^{2}\right]\right)^{1 / 2} \\
& \leq \mathrm{e}^{-\frac{\varepsilon}{2} t}\left(\mathbb{E}\left[\left\|X_{\tau}^{x}-x\right\|_{H}^{2}\right]\right)^{1 / 2} \\
& \quad+\sqrt{2(\alpha+\beta)}\left(\int_{0}^{t} \mathrm{e}^{-\varepsilon(t-s)} \mathbb{E}\left[\left\|P_{1} Y_{s}^{x, \tau}-P_{1} X_{s+\tau}^{x}\right\|_{H}^{2}\right] d s\right)^{1 / 2} \\
& =: I_{1}+I_{2} .
\end{aligned}
$$

The first term $I_{1}$ can be estimated by

$$
I_{1} \leq \mathrm{e}^{-\frac{\varepsilon}{2} t} \sup _{s \in[0,1]}\left(\mathbb{E}\left[\left\|X_{s}^{x}-x\right\|_{H}^{2}\right]\right)^{1 / 2}
$$

To estimate the second term $I_{2}$ we first observe that by condition (A2) we have $P_{1} Y_{s}^{x, \tau}=P_{1} X_{s}^{x}=f(s ; x)$ being deterministic and hence by condition (A3) one has for each $s \geq 0$ that

$$
\begin{aligned}
\mathbb{E}\left[\left\|P_{1} Y_{s}^{x, \tau}-P_{1} X_{s+\tau}^{x}\right\|_{H}^{2}\right] & \leq 2\left\|P_{1} Y_{s}^{x, \tau}-\tilde{f}(x)\right\|_{H}^{2}+2\left\|P_{1} X_{s+\tau}^{x}-\tilde{X}_{\infty}^{x}\right\|_{H}^{2} \\
& \leq 4 C(x) \mathrm{e}^{-\delta(x) s}
\end{aligned}
$$

This readily yields

$$
\begin{aligned}
& \int_{0}^{t} \mathrm{e}^{-\varepsilon(t-s)} \mathbb{E}\left[\left\|P_{1} Y_{s}^{x, \tau}-P_{1} X_{s+\tau}^{x}\right\|_{H}^{2}\right] d s \\
& \quad \leq 4 C(x) \int_{0}^{t} \mathrm{e}^{-\varepsilon(t-s)} \mathrm{e}^{-\delta(x) s} d s \\
& \quad=4 C(x) \mathrm{e}^{-\varepsilon t} \frac{\mathrm{e}^{(\varepsilon-\delta(x)) t}-1}{\varepsilon-\delta(x)} \\
& \quad \leq 4 C(x) \frac{\mathrm{e}^{-\delta(x) t}}{\varepsilon-\delta(x)}
\end{aligned}
$$

Inserting this into the definition of $I_{2}$ gives

$$
I_{2} \leq 2 \sqrt{\frac{(\alpha+\beta) C(x)}{\varepsilon-\delta(x)}} \mathrm{e}^{-\frac{\delta(x)}{2} t} .
$$

Case $\tau>1$ : Fix some $N \in \mathbb{N}$ with $\tau<N<2 \tau$ and define a sequence of numbers $\left(a_{n}\right)_{n=0, \ldots, N}$ by

$$
a_{n}:=\frac{\tau}{N} n, \quad n=0, \ldots, N
$$


Then $a_{0}=0, a_{N}=\tau$ and $a_{n}-a_{n-1}=\frac{\tau}{N}=: \varkappa \in\left(\frac{1}{2}, 1\right)$ for $n=1, \ldots, N$. Hence we obtain from the coupling Lemma 4.7.(b)

$$
\begin{aligned}
\mathrm{W}_{2} & \left(p_{t+\tau}(x, \cdot), p_{t}(x, \cdot)\right) \\
\leq & \sum_{n=1}^{N} \mathrm{~W}_{2}\left(p_{t+a_{n}}(x, \cdot), p_{t+a_{n-1}}(x, \cdot)\right) \\
\leq & \sum_{n=1}^{N}\left(\mathbb { E } \left[\| Y_{t+a_{n-1}}^{x, \varkappa}-X_{\left.\left.t+a_{n-1}+\varkappa \|_{H}^{x}\right]\right)^{1 / 2}}^{N}\right.\right. \\
\leq & \sum_{n=1}^{N} \mathrm{e}^{-\frac{\varepsilon}{2}\left(t+a_{n-1}\right)}\left(\mathbb{E}\left[\left\|X_{\varkappa}^{x}-x\right\|_{H}^{2}\right]\right)^{1 / 2} \\
& +\sqrt{2(\alpha+\beta)} \sum_{n=1}^{N}\left(\int_{0}^{t+a_{n-1}} \mathrm{e}^{-\varepsilon\left(t+a_{n-1}-s\right)} \mathbb{E}\left[\left\|P_{1} Y_{s}^{x, \varkappa}-P_{1} X_{s+\varkappa}^{x}\right\|_{H}^{2}\right] d s\right)^{1 / 2} \\
= & : J_{1}+J_{2} .
\end{aligned}
$$

For the first term $J_{1}$ we use $\varkappa>\frac{1}{2}$ so that

$$
\sum_{n=1}^{N} \mathrm{e}^{-\frac{\varepsilon}{2} \varkappa(n-1)} \leq \sum_{n=0}^{\infty} \mathrm{e}^{-\frac{\varepsilon}{4} n}=\left(1-\mathrm{e}^{-\frac{\varepsilon}{4}}\right)^{-1},
$$

from which we obtain

$$
\begin{aligned}
J_{1} & =\mathrm{e}^{-\frac{\varepsilon}{2} t} \sup _{s \in[0,1]}\left(\mathbb{E}\left[\left\|X_{s}^{x}-x\right\|_{H}^{2}\right]\right)^{\frac{1}{2}} \sum_{n=1}^{N} \mathrm{e}^{-\frac{\varepsilon}{2} \varkappa(n-1)} \\
& \leq \sup _{s \in[0,1]}\left(\mathbb{E}\left[\left\|X_{s}^{x}-x\right\|_{H}^{2}\right]\right)^{\frac{1}{2}}\left(1-\mathrm{e}^{-\frac{\varepsilon}{4}}\right)^{-1} \mathrm{e}^{-\frac{\varepsilon}{2} t} .
\end{aligned}
$$

To estimate the second term $J_{2}$ we first observe that by condition (A2) we have $P_{1} Y_{s}^{x, \tau}=P_{1} X_{s}^{x}=f(s ; x)$ being deterministic and hence by condition (A3), one has for $s \geq 0$

$$
\begin{aligned}
\mathbb{E}\left[\left\|P_{1} Y_{s}^{x, \varkappa}-P_{1} X_{s+\varkappa}^{x}\right\|_{H}^{2}\right] & \leq 2\left\|P_{1} Y_{s}^{x, \varkappa}-\tilde{f}(x)\right\|_{H}^{2}+2\left\|P_{1} X_{s+\varkappa}^{x}-\tilde{f}(x)\right\|_{H}^{2} \\
& \leq 4 C(x) \mathrm{e}^{-\delta(x) s} .
\end{aligned}
$$

Hence we find that

$$
\begin{aligned}
& \int_{0}^{t+a_{n-1}} \mathrm{e}^{-\varepsilon\left(t+a_{n-1}-s\right)} \mathbb{E}\left[\left\|P_{1} Y_{s}^{x, \varkappa}-P_{1} X_{s+\varkappa}^{x}\right\|_{H}^{2}\right] d s \\
& \quad \leq 4 C(x) \int_{0}^{t+a_{n-1}} \mathrm{e}^{-\varepsilon\left(t+a_{n-1}-s\right)} \mathrm{e}^{-\delta(x) s} d s \\
& \quad=4 C(x) \mathrm{e}^{-\varepsilon\left(t+a_{n-1}\right)} \frac{\mathrm{e}^{(\varepsilon-\delta(x))\left(t+a_{n-1}\right)}-1}{\varepsilon-\delta(x)} \\
& \leq 4 C(x) \frac{\mathrm{e}^{-\delta(x) t}}{\varepsilon-\delta(x)} \mathrm{e}^{-\delta(x) a_{n-1}} \\
& \quad \leq 4 C(x) \frac{\mathrm{e}^{-\delta(x) t}}{\varepsilon-\delta(x)} \mathrm{e}^{-\frac{\delta(x)}{2}(n-1)}
\end{aligned}
$$


where the last inequality follows from $a_{n-1}=\varkappa(n-1) \geq \frac{1}{2}(n-1)$. From this we readily derive the estimate

$$
J_{2} \leq 2 \sqrt{\frac{(\alpha+\beta) C(x)}{\varepsilon-\delta(x)}}\left(1-\mathrm{e}^{-\frac{\delta(x)}{4}}\right)^{-1} \mathrm{e}^{-\frac{\delta(x)}{2} t} .
$$

Hence, using also (3.5) we obtain

$$
\mathrm{W}_{2}\left(p_{t+\tau}(x, \cdot), p_{t}(x, \cdot)\right) \leq K(\alpha, \beta, \varepsilon, x) \mathrm{e}^{-\frac{\delta(x)}{2} t}, \quad t, \tau \geq 0,
$$

where the constant $K(\alpha, \beta, \varepsilon, x)>0$ is given by

$$
K(\alpha, \beta, \varepsilon, x)=K(\varepsilon)\left(1+\|x\|_{H}\right)+2 \sqrt{\frac{(\alpha+\beta) C(x)}{\varepsilon-\delta(x)}}\left(1-\mathrm{e}^{-\frac{\delta(x)}{4}}\right)^{-1}
$$

with another constant $K(\varepsilon)>0$. This implies that, for each $x \in H,\left(p_{t}(x, \cdot)\right)_{t \geq 0}$ has a limit in $\mathcal{P}_{2}(H)$. Denote this limit by $\pi_{\delta_{x}}$. Assertion (a) now follows by taking the limit $\tau \rightarrow \infty$ in (4.10) and using the fact that $K(\alpha, \beta, \varepsilon, x)$ is independent of $\tau$.

It remains to prove assertion (b). First observe that, using $\delta(x) \geq \delta>0$ and $C(x) \leq C\left(1+\|x\|_{H}\right)^{4}$, we have

$$
K(\alpha, \beta, \varepsilon, x) \leq\left(1+\|x\|_{H}\right)^{2} \widetilde{K}(\alpha, \beta, \varepsilon)
$$

for some constant $\widetilde{K}(\alpha, \beta, \varepsilon)$. Note that

$$
p_{t}^{*} \rho(d y)=\int_{H} p_{t}(z, d y) \rho(d z) \quad \text { and } \quad p_{t+\tau}^{*} \rho(d y)=\int_{H} p_{t+\tau}(z, d y) \rho(d z) .
$$

Hence using first the convexity of the Wasserstein distance and then (4.10) we find that

$$
\begin{aligned}
\mathrm{W}_{2}\left(p_{t+\tau}^{*} \rho, p_{t}^{*} \rho\right) & \leq \int_{H} \mathrm{~W}_{2}\left(p_{t+\tau}(x, \cdot), p_{t}(x, \cdot)\right) \rho(d x) \\
& \leq \widetilde{K}(\alpha, \beta, \varepsilon) \int_{H}\left(1+\|x\|_{H}\right)^{2} \rho(d x) \cdot \mathrm{e}^{-\frac{\delta}{2} t} .
\end{aligned}
$$

Since $\rho \in \mathcal{P}_{2}(H)$, the assertion is proved.

\subsection{Proof of Corollary 4.4}

Proof of Corollary 4.4. Recall that, by condition (A2) the process $P_{1} X_{t}^{x}$ solves

$$
P_{1} X_{t}^{x}=P_{1} S(t) P_{1} x+\int_{0}^{t} P_{1} S(t-s) F\left(P_{1} X_{s}^{x}\right) d s .
$$

Since $F$ is globally Lipschitz continuous by condition (A1), it follows that this equation has for each $x \in H$ a unique solution and is deterministic. From this we readily conclude that $P_{1} X_{t}^{x}=P_{1} X_{t}^{y}$ holds for all $t \geq 0$, provided that $P_{1} x=P_{1} y$. Hence Proposition 3.5 yields for such $x, y$

$$
\mathbb{E}\left[\left\|X_{t}^{x}-X_{t}^{y}\right\|_{H}^{2}\right] \leq \mathrm{e}^{-\varepsilon t}\|x-y\|_{H}^{2}, \quad \forall t \geq 0
$$


Then for each $x, y \in H$ with $P_{1} x=P_{1} y$ and each $t \geq 0$ we obtain

$$
\begin{aligned}
\mathrm{W}_{2}\left(\pi_{\delta_{x}}, \pi_{\delta_{y}}\right) & \leq \mathrm{W}_{2}\left(\pi_{\delta_{x}}, p_{t}(x, \cdot)\right)+\mathrm{W}_{2}\left(p_{t}(x, \cdot), p_{t}(y, \cdot)\right)+\mathrm{W}_{2}\left(p_{t}(y, \cdot), \pi_{\delta_{y}}\right) \\
& \leq \mathrm{W}_{2}\left(\pi_{\delta_{x}}, p_{t}(x, \cdot)\right)+\mathrm{e}^{-\frac{\varepsilon}{2} t}\|x-y\|_{H}+\mathrm{W}_{2}\left(p_{t}(y, \cdot), \pi_{\delta_{y}}\right) .
\end{aligned}
$$

Letting $t \rightarrow \infty$ yields $\pi_{\delta_{x}}=\pi_{\delta_{y}}$ and hence assertion (a) is proved.

To prove assertion (b), let $\rho, \widetilde{\rho} \in \mathcal{P}_{2}(H)$ be such that $\rho \circ P_{1}^{-1}=\widetilde{\rho} \circ P_{1}^{-1}$. Then

$$
\mathrm{W}_{2}\left(\pi_{\rho}, \pi_{\tilde{\rho}}\right) \leq \mathrm{W}_{2}\left(\pi_{\rho}, p_{t}^{*} \rho\right)+\mathrm{W}_{2}\left(p_{t}^{*} \rho, p_{t}^{*} \widetilde{\rho}\right)+\mathrm{W}_{2}\left(p_{t}^{*} \widetilde{\rho}, \pi_{\tilde{\rho}}\right)
$$

Again, by letting $t \rightarrow \infty$, it suffices to prove that

$$
\limsup _{t \rightarrow \infty} \mathrm{W}_{2}\left(p_{t}^{*} \rho, p_{t}^{*} \widetilde{\rho}\right)=0 \text {. }
$$

Let $G$ be a coupling of $(\rho, \widetilde{\rho})$. Using the convexity of the Wasserstein distance and Proposition 3.5 gives

$$
\begin{aligned}
\mathrm{W}_{2} & \left(p_{t}^{*} \rho, p_{t}^{*} \widetilde{\rho}\right) \\
\leq & \int_{H \times H} \mathrm{~W}_{2}\left(p_{t}(x, \cdot), p_{t}(y, \cdot)\right) G(d x, d y) \\
\leq & \int_{H \times H}\left(\mathbb{E}\left[\left\|X_{t}^{x}-X_{t}^{y}\right\|_{H}^{2}\right]\right)^{1 / 2} G(d x, d y) \\
\leq & \int_{H \times H} \mathrm{e}^{-\frac{\varepsilon}{2} t}\|x-y\|_{H} G(d x, d y) \\
& +\sqrt{2(\alpha+\beta)} \int_{H \times H}\left(\int_{0}^{t} \mathrm{e}^{-\varepsilon(t-s)} \mathbb{E}\left[\left\|P_{1} X_{s}^{x}-P_{1} X_{s}^{y}\right\|_{H}^{2}\right] d s\right)^{1 / 2} G(d x, d y) \\
= & : I_{1}+I_{2} .
\end{aligned}
$$

The first term $I_{1}$ satisfies

$$
I_{1} \leq\left(2+\int_{H}\|x\|_{H}^{2} \rho(d x)+\int_{H}\|y\|_{H}^{2} \widetilde{\rho}(d y)\right) \mathrm{e}^{-\frac{\varepsilon}{2} t} .
$$

For the second term we first use (A2) so that $P_{1} X_{s}^{x}=P_{1} X_{s}^{P_{1} x}, P_{1} X_{s}^{y}=$ $P_{1} X_{s}^{P_{1} y}$ and hence we find for each $T>0$ a constant $C(T)>0$ such that for $t \in[0, T]$

$$
\begin{aligned}
I_{2} & =\sqrt{2(\alpha+\beta)} \int_{H_{1} \times H_{1}}\left(\int_{0}^{t} \mathrm{e}^{-\varepsilon(t-s)}\left\|P_{1} X_{s}^{x}-P_{1} X_{s}^{y}\right\|_{H}^{2} d s\right)^{1 / 2} G(d x, d y) \\
& \leq C(T)\left(\int_{H \times H}\left\|P_{1} x-P_{1} y\right\|_{H}^{2} G(d x, d y)\right)^{1 / 2} .
\end{aligned}
$$

Let us choose a particular coupling $G$ as follows: By disintegration we write $\rho(d x)=\rho\left(x_{1}, d x_{0}\right)\left(\rho \circ P_{1}^{-1}\right)\left(d x_{1}\right), \widetilde{\rho}(d x)=\widetilde{\rho}\left(x_{1}, d x_{0}\right)\left(\widetilde{\rho} \circ P_{1}^{-1}\right)\left(d x_{1}\right)=$ $\widetilde{\rho}\left(x_{1}, d x_{0}\right)\left(\rho \circ P_{1}^{-1}\right)\left(d x_{1}\right)$ where $\rho\left(x_{1}, d x_{0}\right), \widetilde{\rho}\left(x_{1}, d x_{0}\right)$ are conditional probabilities defined on $\mathcal{B}\left(H_{0}\right)$ and we have used that $\left(\rho \circ P_{1}^{-1}\right)\left(d x_{1}\right)=\left(\widetilde{\rho} \circ P_{1}^{-1}\right)\left(d x_{1}\right)$. Then $G$ is, for $A, B \in \mathcal{B}(H)$, given by

$$
G(A \times B):=\int_{H \times H} \mathbb{1}_{A}\left(x_{0}, x_{1}\right) \mathbb{1}_{B}\left(y_{0}, y_{1}\right) \rho\left(x_{1}, d x_{0}\right) \widetilde{\rho}\left(y_{1}, d y_{0}\right) \widetilde{G}\left(d x_{1}, d y_{1}\right),
$$


where $\widetilde{G}$ is a probability measure on $H_{1}^{2}$ given, for $A_{1}, B_{1} \in \mathcal{B}\left(H_{1}\right)$, by

$$
\widetilde{G}\left(A_{1} \times B_{1}\right)=\left(\rho \circ P_{1}^{-1}\right)\left(A_{1} \cap B_{1}\right)=\rho\left(\left\{x \in H \mid P_{1} x \in A_{1} \cap B_{1}\right\}\right) .
$$

For this particular choice of $G$ we find that

$$
\begin{aligned}
\int_{H \times} & \left\|P_{1} x-P_{1} y\right\|_{H}^{2} G(d x, d y) \\
= & \int_{H_{1} \times H_{1}} \int_{H_{0}^{2}}\left\|x_{1}-y_{1}\right\|_{H}^{2} \rho\left(x_{1}, d x_{0}\right) \widetilde{\rho}\left(y_{1}, d y_{0}\right) \widetilde{G}\left(d x_{1}, d y_{1}\right) \\
= & \int_{H_{1} \times H_{1}}\left\|x_{1}-y_{1}\right\|_{H}^{2} \widetilde{G}\left(d x_{1}, d y_{1}\right)=0
\end{aligned}
$$

and hence $I_{2}=0$, since $\widetilde{G}$ is supported on the diagonal of $H_{1} \times H_{1}$. This proves (4.12) and completes the proof.

\section{The Heath-Jarrow-Mortion-Musiela equation}

The Heath-Jarrow-Morton-Musiela equation (HJMM-equation) describes the term structure of interest rates in terms of its forward rate dynamics modelled, for $\beta>0$ fixed, on the separable Hilbert space of forward curves

$$
\begin{aligned}
H_{\beta} & =\left\{h: \mathbb{R}_{+} \rightarrow \mathbb{R}: h \text { is absolutely continuous and }\|h\|_{\beta}<\infty\right\}, \\
\langle h, g\rangle_{\beta} & =h(\infty) g(\infty)+\int_{0}^{\infty} h^{\prime}(x) g^{\prime}(x) \mathrm{e}^{\beta x} d x
\end{aligned}
$$

with norm $\|h\|_{\beta}^{2}=\langle h, h\rangle_{\beta}$. Such space was first motivated and introduced by Filipovic [15]. Note that $h(\infty):=\lim _{x \rightarrow \infty} h(x)$ exists, whenever $\int_{0}^{\infty}\left(h^{\prime}(x)\right)^{2}$ $\mathrm{e}^{\beta x} d x<\infty$. It is called the long rate of the forward curve $h$. The HJMMequation on $H_{\beta}$ is given by

$$
\left\{\begin{array}{l}
d X_{t}=\left(A X_{t}+F_{H J M M}(\sigma, \gamma)\left(X_{t}\right)\right) d t+\sigma\left(X_{t}\right) d W_{t}+\int_{E} \gamma\left(X_{t}, \nu\right) \tilde{N}(d t, d \nu) \\
X_{0} \in L^{2}\left(\Omega, \mathcal{F}_{0}, \mathbb{P} ; H_{\beta}\right)
\end{array}\right.
$$

where $\left(W_{t}\right)_{t \geq 0}$ is a $Q$-Wiener process, $\tilde{N}(d t, d \nu)$ is a compensated Poisson random measure on $E$ with compensator $d t \mu(d \nu)$ as defined in Sect. 3.1 for $H:=H_{\beta}$, and

(i) $A$ is the infinitesimal generator of the shift semigroup $(S(t))_{t \in \mathbb{R}_{+}}$on $H_{\beta}$, that is $S(t) h(x):=h(x+t)$ for all $t, x \geq 0$.

(ii) $h \mapsto \sigma(h)$ is a $\mathcal{B}\left(H_{\beta}\right) / \mathcal{B}\left(L_{2}^{0}\right)$-measurable mapping from $H_{\beta}$ into $L_{2}^{0}\left(H_{\beta}\right)$ and $(h, \nu) \mapsto \gamma(h, \nu)$ is $\mathcal{B}\left(H_{\beta}\right) \otimes \mathcal{E} / \mathcal{B}\left(H_{\beta}\right)$-measurable mapping from $H_{\beta} \times$ $E$ into $H_{\beta}$.

(iii) The drift is of the form

$$
F_{H J M M}(\sigma, \gamma)(h)=\sum_{j \in \mathbb{N}} \sigma^{j}(h) \Sigma^{j}(h)-\int_{E} \gamma(h, \nu)\left(\mathrm{e}^{\Gamma(h, \nu)}-1\right) \mu(d \nu),
$$




$$
\begin{aligned}
\text { with } \sigma^{j}(h) & =\sqrt{\lambda_{j}} \sigma(h) e_{j}, \\
\Sigma^{j}(h)(t) & =\int_{0}^{t} \sigma^{j}(h)(s) d s \text { and } \Gamma(h, \nu)(t)=-\int_{0}^{t} \gamma(h, \nu)(s) d s .
\end{aligned}
$$

The special form of the drift stems from mathematical finance and is sufficient for the absence of arbitrage opportunities. We denote the space of all forward rates with long rate equal to zero by

$$
H_{\beta}^{0}=\left\{h \in H_{\beta}: h(\infty)=0\right\} .
$$

For the construction of a unique mild solution to (5.2) the following conditions have been introduced in [11]:

(B1) $\sigma: H_{\beta} \rightarrow L_{2}^{0}\left(H_{\beta}^{0}\right), \gamma: H_{\beta} \times E \rightarrow H_{\beta^{\prime}}^{0}$ are Borel measurable for some $\beta^{\prime}>\beta$.

(B2) There exists a function $\Phi: E \rightarrow \mathbb{R}_{+}$such that $\Phi(\nu) \geq|\Gamma(h, \nu)(t)|$ for all $h \in H_{\beta}, \nu \in E$ and $t \geq 0$.

(B3) There is an $M \geq 0$ such that, for all $h \in H_{\beta}$, and some $\beta^{\prime}>\beta$

$$
\|\sigma(h)\|_{L_{2}^{0}\left(H_{\beta}\right)} \leq M, \quad \int_{E} \mathrm{e}^{\Phi(\nu)} \max \left\{\|\gamma(h, \nu)\|_{\beta^{\prime}}^{2},\|\gamma(h, \nu)\|_{\beta^{\prime}}^{4}\right\} \mu(d \nu) \leq M .
$$

(B4) The function $F_{2}: H_{\beta} \rightarrow H_{\beta}^{0}$ defined by

$$
F_{2}(h)=-\int_{E} \gamma(h, \nu)\left(\mathrm{e}^{\Gamma(h, \nu)}-1\right) \mu(d \nu)
$$

has the weak derivative given by

$$
\frac{d}{d x} F_{2}(h)=\int_{E} \gamma(h, \nu)^{2} \mathrm{e}^{\Gamma(h, \nu)} \mu(d \nu)-\int_{E}\left(\frac{d}{d x} \gamma(h, \nu)\right)\left(\mathrm{e}^{\Gamma(h, \nu)}-1\right) \mu(d \nu) .
$$

(B5) There are constants $L_{\sigma}, L_{\gamma}>0$ such that, for all $h_{1}, h_{2} \in H_{\beta}$, we have

$$
\begin{aligned}
& \left\|\sigma\left(h_{1}\right)-\sigma\left(h_{2}\right)\right\|_{L_{2}^{0}\left(H_{\beta}\right)}^{2} \leq L_{\sigma}\left\|h_{1}-h_{2}\right\|_{\beta}^{2}, \\
& \int_{E} \mathrm{e}^{\Phi(\nu)}\left\|\gamma\left(h_{1}, \nu\right)-\gamma\left(h_{2}, \nu\right)\right\|_{\beta^{\prime}}^{2} \mu(d \nu) \leq L_{\gamma}\left\|h_{1}-h_{2}\right\|_{\beta}^{2} .
\end{aligned}
$$

The following is the basic existence and uniqueness result for the HeathJarrow-Morton-Musiela equation (5.2).

Theorem 5.1. [11] Suppose that conditions (B1)-(B5) are satisfied. Then $F_{H J M M}: H_{\beta} \longrightarrow H_{\beta}^{0}$ and there exists a constant $L_{F}>0$ such that, for each $h_{1}, h_{2} \in H_{\beta}$,

$$
\left\|F_{H J M M}\left(h_{1}\right)-F_{H J M M}\left(h_{2}\right)\right\|_{\beta}^{2} \leq L_{F}\left\|h_{1}-h_{2}\right\|_{\beta}^{2} .
$$

This constant can be chosen as

$$
L_{F}=\frac{\max \left(L_{\sigma}, L_{\gamma}\right) \sqrt{M}}{\beta}\left(\sqrt{6 M \sqrt{2}}+\sqrt{\frac{8}{\beta^{3}}+\frac{16}{\beta}}+\sqrt{\frac{16\left(1+\frac{1}{\sqrt{\beta}}\right)^{2}+48}{\left(\beta^{\prime}-\beta\right)}}\right) .
$$

Moreover, for each initial condition $h \in L^{2}\left(\Omega, \mathcal{F}_{0}, \mathbb{P} ; H_{\beta}\right)$ there is a unique adapted, cádlág mild solution $\left(X_{t}\right)_{t \geq 0}$ to $(5.2)$. 
Proof. This result can be found essentially in [11], where the bound on $L_{F}$ is an immediate result from its derivation. position

Using the space of all functions with zero long rate we obtain the decom-

$$
H_{\beta}=H_{\beta}^{0} \oplus \mathbb{R}, \quad h=(h-h(\infty))+h(\infty),
$$

where $h(\infty) \in \mathbb{R}$ is identified with a constant function. Denote by

$$
P_{0} h=h-h(\infty) \text { and } P_{1} h=h(\infty)
$$

the corresponding projections onto $H_{\beta}^{0}$ and $\mathbb{R}$, respectively. Such a decomposition of $H_{\beta}$ was first used in [38] to study invariant measures for the HJMM-equation driven by a $Q$-Wiener process. An extension to the Lévy driven HJMM-equation was then obtained in [36]. The proof of the next theorem shows that the results of Sect. 4 imply the stability properties of the HJMM-equation as a particular case.

Theorem 5.2. Suppose that conditions (B1)-(B5) are satisfied. If

$$
\beta>2 \sqrt{L_{F}}+L_{\sigma}+L_{\gamma},
$$

then for each initial distribution $\rho$ on $H_{\beta}$ with finite second moments there exists an invariant measure $\pi_{\rho}$ and it holds that

$$
\mathrm{W}_{2}\left(p_{t}^{*} \rho, \pi_{\rho}\right) \leq K\left(1+\int_{H_{\beta}}\|h\|_{\beta}^{2} \rho(d h)\right) \mathrm{e}^{-\frac{\beta-2 \sqrt{L_{F}}-L_{\sigma}-L_{\gamma}}{2} t}
$$

for some constant $K=K(\beta, \sigma, \gamma)>0$. Moreover, given $\rho, \widetilde{\rho}$ such that $\rho \circ P_{1}^{-1}=$ $\widetilde{\rho} \circ P_{1}^{-1}$, then $\pi_{\rho}=\pi_{\widetilde{\rho}}$.

Proof. Observe that the assertion is an immediate consequence of Theorem 4.3 and Corollary 4.4. Below we briefly verify the assumptions given in these statements. Condition (A1) follows from (B1), (B5), and (5.3). The growth condition (3.14) is satisfied by (B3) and the fact that $\|\cdot\|_{\beta} \leq\|\cdot\|_{\beta^{\prime}}$ for $\beta<\beta^{\prime}$. It is not difficult to see that

$$
\left\|S(t) h-P_{1} h\right\|_{\beta} \leq \mathrm{e}^{-\frac{\beta}{2} t}\left\|h-P_{1} h\right\|_{\beta}, \quad t \geq 0
$$

and that $(S(t))_{t \geq 0}$ leaves $H_{\beta}^{0}$ as well as $\mathbb{R} \subset H_{\beta}$ invariant. Hence Remark 3.2 yields that

$$
\langle A h, h\rangle_{\beta} \leq-\frac{\beta}{2}\|h\|_{\beta}^{2}+\frac{\beta}{2}\left\|P_{1} h\right\|_{\beta}^{2}, \quad h \in D(A) .
$$

It follows from the considerations in Sect. 2 (see (3.10)) that (GDC) is satisfied for $\alpha=\frac{\beta}{2}-\sqrt{L_{F}}$. Consequently, $\varepsilon=\beta-2 \sqrt{L_{F}}-L_{\sigma}-L_{\gamma}$ and (3.13) holds due to (5.5). Since the coefficients map into $H_{\beta}^{0}$ and $S(t) P_{1} h=h(\infty)=P_{1} h$, conditions (A2), (A3) and (4.6) are trivially satisfied. The particular form of the estimate (5.6) follows from the proof of Theorem 4.3. 
Comparing our result with $[36,38]$, we allow for a more general jump noise and prove convergence in the stronger Wasserstein distance with an exponential rate. Moreover, assuming that the volatilities map constant functions onto zero, i.e.

$$
\sigma(c) \equiv 0, \quad \gamma(c, \nu) \equiv 0, \quad \forall c \in \mathbb{R} \subset H_{\beta}, \nu \in E
$$

shows that $F(c) \equiv 0$ and hence also (4.4) is satisfied. Hence we may apply Theorem 4.2 to characterize these invariant measures more explicitly. In fact, since $P_{1} h=h(\infty)$ holds for all $h \in H_{\beta}$ we get the following corollary.

Corollary 5.3. Suppose that conditions (B1) - (B5) are satisfied, that (5.5) and (5.7) hold. Then

$$
\mathbb{E}\left[\left\|X_{t}-X_{0}(\infty)\right\|_{\beta}^{2}\right] \leq \mathbb{E}\left[\left\|X_{0}-X_{0}(\infty)\right\|_{\beta}^{2}\right] \mathrm{e}^{-\left(\beta-2 \sqrt{L_{F}}-L_{\sigma}-L_{\gamma}\right) t}
$$

for each $X_{0} \in L^{2}\left(\Omega, \mathcal{F}_{0}, \mathbb{P} ; H_{\beta}\right)$.

This Corollary describes a case where the set of multiple invariant measures is explicitly given by the laws of square integrable random variables over the continuum of long rates, including the case of invariant measures $\delta_{h_{0}(\infty)}$ for all $X_{0}=h_{0} \in H_{\beta}$.

We close this section by applying our results for the particular example discussed before also in [36].

Example 5.4. Take

$$
\sigma^{1}(h)(x):=\int_{x}^{\infty} \min \left(\mathrm{e}^{-\beta y},\left|h^{\prime}(y)\right|\right) d y
$$

and $\sigma^{j} \equiv 0$ for $j \geq 2$. Then

$$
\|\sigma(h)\|_{L_{2}^{0}\left(H_{\beta}\right)}^{2}=\left\|\sigma^{1}(h)\right\|_{\beta}^{2} \leq \int_{0}^{\infty}\left(\mathrm{e}^{-2 \beta x}\right) \mathrm{e}^{\beta x} d x=\frac{1}{\beta}=: M
$$

and since $\min \left(a, b_{1}\right)-\min \left(a, b_{2}\right) \leq\left|b_{1}-b_{2}\right|$ for $a, b_{1}, b_{2} \in \mathbb{R}_{+}$, we also have

$$
\begin{aligned}
\left\|\sigma\left(h_{1}\right)-\sigma\left(h_{2}\right)\right\|_{L_{2}^{0}\left(H_{\beta}\right)}^{2} & =\left\|\sigma^{1}\left(h_{1}\right)-\sigma^{1}\left(h_{2}\right)\right\|_{\beta}^{2} \\
& =\int_{0}^{\infty}\left(\min \left(\mathrm{e}^{-\beta x},\left|h_{1}^{\prime}(x)\right|\right)-\min \left(\mathrm{e}^{-\beta x},\left|h_{2}^{\prime}(x)\right|\right)\right)^{2} \mathrm{e}^{\beta x} d x \\
& \leq \int_{0}^{\infty}\left(h_{1}^{\prime}(x)-h_{2}^{\prime}(x)\right)^{2} \mathrm{e}^{\beta x} d x \\
& \leq\left\|h_{1}-h_{2}\right\|_{\beta}^{2} .
\end{aligned}
$$

Consequently, by taking $\gamma \equiv 0$, the conditions (B1) - (B5) are satisfied with $L_{\sigma}=1$ and $L_{\gamma}=0$ and $M=\frac{1}{\beta}$ for the Lipschitz and growth constants. By (5.4) we get

$$
L_{F}=\frac{1}{\sqrt{\beta^{3}}}\left(\sqrt{\frac{6 \sqrt{2}}{\beta}}+\sqrt{\frac{8}{\beta^{3}}+\frac{16}{\beta}}+\sqrt{\frac{16\left(1+\frac{1}{\sqrt{\beta}}\right)^{2}+48}{\left(\beta^{\prime}-\beta\right)}}\right),
$$


for $\beta^{\prime}>\beta$. Choosing $\beta \geq 3$ and $\beta^{\prime}>\beta$ large enough such that $L_{F}<1$, we find that

$$
2 \sqrt{L_{F}}+L_{\sigma}+L_{\gamma}<3=\beta,
$$

i.e. (5.5) is satisfied. It is clear that $\sigma(c) \equiv 0$ for each constant function $c$. Hence Corollary 5.3 is applicable.

\section{Stochastic partial differential equations with delay}

\subsection{Description of the model}

Let $H$ be a separable Hilbert space and $\left(W_{t}\right)_{t>0}$ a $Q$-Wiener process on a stochastic basis $\left(\Omega, \mathcal{F},\left(\mathcal{F}_{t}\right)_{t \geq 0}, \mathbb{P}\right)$ with the usual conditions. Below we investigate invariant measures for the stochastic delay equation

$$
\left\{\begin{array}{l}
d X_{t}=\left(A X_{t}+G\left(X_{t+\cdot}\right)\right) d t+\sigma\left(X_{t}, X_{t+\cdot}\right) d W_{t}, \quad t>0 \\
X_{0}=\phi_{0}, X_{0+\cdot}=\phi
\end{array}\right.
$$

where $\phi_{0} \in L^{2}\left(\Omega, \mathcal{F}_{0}, \mathbb{P} ; H\right), \phi \in L^{2}\left(\Omega, \mathcal{F}_{0}, \mathbb{P} ; L^{2}([-1,0] ; H)\right)$ and for $t \geq 1$ the term $X_{t+}$. denotes the past segment of the trajectory, i.e.

$$
\begin{aligned}
X_{t+\cdot}:[-1,0] & \longrightarrow H \\
s & \longmapsto X_{t+s},
\end{aligned}
$$

and for $t \in[0,1)$

$$
\begin{aligned}
X_{t+\cdot}:[-1,0] & \longrightarrow H \\
s & \longmapsto \phi(t+s) \mathbf{1}_{[-1,-t)}(s)+X_{t+s} \mathbf{1}_{[-t, 0]}(s),
\end{aligned}
$$

and

(i) $(A, D(A))$ is the infinitesimal generator of a strongly continuous semigroup $(S(t))_{t \geq 0}$ on $H$.

(ii) $\left(\psi_{0}, \psi\right) \mapsto \sigma\left(\bar{\psi}_{0}, \psi\right)$ is measurable from $H \times L^{2}([-1,0] ; H)$ to $L_{2}^{0}(H)$.

(iii) $G: W^{1,2}([-1,0] ; H) \rightarrow H$ is a continuous linear operator given by the Riemann-Stieltjes integral

$$
G \phi:=\int_{-1}^{0} \eta(d s) \phi(s)
$$

where $\eta:[-1,0] \rightarrow L(H)$ is of bounded variation.

Such an equation is usually studied in an extended Hilbert space which also takes the evolution of the past segment $\left(X_{t+} \cdot\right)_{t \geq 0}$ into account, see [8]. Below we follow this approach. Namely, introduce the new Hilbert space

$$
\mathcal{H}=H \times L^{2}([-1,0] ; H), \quad\left\|\left(\phi_{0}, \phi\right)\right\|_{\mathcal{H}}=\left(\left\|\phi_{0}\right\|_{H}^{2}+\|\phi\|_{L^{2}([-1,0] ; H)}^{2}\right)^{1 / 2} .
$$

Define the operator

$$
\mathcal{A}_{0}:=\left(\begin{array}{cc}
A & 0 \\
0 & \frac{d}{d s}
\end{array}\right) \quad D(\mathcal{A})=\left\{\left(\phi_{0}, \phi\right)^{T} \in D(A) \times W^{1,2}([0,1] ; H): \phi(0)=\phi_{0}\right\}
$$


which generates a strongly continuous semigroup $\left(\mathcal{S}_{0}(t)\right)_{t \geq 0}$ on $\mathcal{H}$, given by

$$
\mathcal{S}_{0}(t):=\left(\begin{array}{cc}
S(t) & 0 \\
S_{t} & T_{0}(t)
\end{array}\right)
$$

due to [5, Theorem 3.25]. Here $\left(T_{0}(t)\right)_{t \geq 0}$ is the nilpotent left shift semigroup on $L^{2}([-1,0] ; H)$ and

$$
S_{t} \phi_{0}(\tau):= \begin{cases}S(t+\tau) \phi_{0}, & -t<\tau \leq 0 \\ 0, & -1 \leq \tau \leq-t .\end{cases}
$$

It then follows from [5, Theorem 3.29] that the operator $\mathcal{A}$ with domain $D(\mathcal{A})=D\left(\mathcal{A}_{0}\right)$ given by

$$
\mathcal{A}:=\left(\begin{array}{cc}
A & G \\
0 & \frac{d}{d s}
\end{array}\right)=\mathcal{A}_{0}+\left(\begin{array}{cc}
0 & G \\
0 & 0
\end{array}\right)
$$

is the generator of a strongly continuous semigroup $(\mathcal{S}(t))_{t \geq 0}$ on $\mathcal{H}$. Thus, we can formally identify (6.1) with the $\mathcal{H}$-valued SPDE

$$
\left\{\begin{array}{l}
d \mathcal{X}_{t}=\mathcal{A} \mathcal{X}_{t} d t+\Sigma\left(\mathcal{X}_{t}\right) d W_{t} \\
\mathcal{X}_{0}=\left(\phi_{0}, \phi\right)^{T} \quad t \geq 0,
\end{array} \quad \Sigma\left(\phi_{0}, \phi\right):=\left(\begin{array}{cc}
\sigma\left(\phi_{0}, \phi\right) & 0 \\
0 & 0
\end{array}\right) .\right.
$$

\subsection{Main results for $(6.5)$}

Next we proceed to apply the results of this work to the SPDE (6.5). For this purpose we make the following assumption:

(C1) There exists an $L_{\sigma}>0$ such that

$$
\left\|\sigma\left(\phi_{0}, \phi\right)-\sigma\left(\psi_{0}, \psi\right)\right\|_{L_{0}^{2}(H)}^{2} \leq L_{\sigma}\left(\left\|\phi_{0}-\psi_{0}\right\|_{H}^{2}+\|\phi-\psi\|_{L^{2}([-1,0] ; H)}^{2}\right)
$$

holds for all $\left(\phi, \phi_{0}\right),\left(\psi_{0}, \psi\right) \in \mathcal{H}$.

(C2) The operator $(A, D(A))$ satisfies (GDC) with projection operators $P_{0}, P_{1}$ and constants $\alpha>0, \beta \geq 0$.

We will see that condition (C1) implies (A1), condition (C2) will be used to prove that $\mathcal{A}$ also satisfies (GDC) with respect to a (possibly equivalent) scalar product on $\mathcal{H}$.

Proposition 6.1. Suppose that conditions (C1), (C2) are satisfied, that $\eta$ has a jump at -1 and that one of the following conditions hold:

(i) $G$ is bounded on $L^{2}([-1,0] ; H)$, z or

(ii) $(S(t))_{t \geq 0}$ leaves $H_{0}=\operatorname{ran}\left(P_{0}\right)$ and $H_{1}=\operatorname{ran}\left(P_{1}\right)$ invariant, $H_{0}, H_{1}$ are orthogonal, $\operatorname{ran}(G) \subset H_{1}$ and $G P_{0}$ extends to a bounded linear operator on $L^{2}([-1,0] ; H)$.

Then for each initial condition $\left(\phi_{0}, \phi\right) \in L^{2}\left(\Omega, \mathcal{F}_{0}, \mathbb{P} ; \mathcal{H}\right)$ there exists a unique mild solution $\left(\mathcal{X}_{t}\right)_{t \geq 0} \subset L^{2}(\Omega, \mathcal{F}, \mathbb{P} ; \mathcal{H})$ to $(6.5)$. 
Proof. Under condition (i) we work on the Hilbert space $\mathcal{H}$ while under condition (ii) we work on the Hilbert space $\mathcal{H}^{\tau}$, which is algebraically $\mathcal{H}$ but equipped with the equivalent norm given by

$$
\left\|\left(\phi_{0}, \phi\right)\right\|_{\mathcal{H}^{\tau}}^{2}:=\left\|\phi_{0}\right\|_{H}^{2}+\int_{-1}^{0}\left\|P_{0} \phi(s)\right\|_{H}^{2} d s+\int_{-1}^{0}\left\|P_{1} \phi(s)\right\|_{H}^{2} \tau(s) d s,
$$

where

$$
\tau(r)=\int_{-1}^{r}\|\eta(d r)\|_{L(H)}, \quad r \in[-1,0]
$$

denotes the variation of $\eta$. Note that due to a result of Webb (see [41] and Remark 6.4 below) this norm is, indeed, equivalent to the original norm on $\mathcal{H}$. For condition (A1) we first observe that $L_{F}=L_{\gamma}=0$ (as $\left.F=0, \gamma=0\right)$ and if assumption (i) holds, then

$$
\begin{aligned}
\left\|\Sigma\left(\phi_{0}, \phi\right)-\Sigma\left(\psi_{0}, \psi\right)\right\|_{L_{0}^{2}(\mathcal{H})}^{2} & \leq\left\|\sigma\left(\phi_{0}, \phi\right)-\sigma\left(\psi_{0}, \psi\right)\right\|_{L_{0}^{2}(H)}^{2} \\
& \leq L_{\sigma}\left(\left\|\phi_{0}-\psi_{0}\right\|_{H}^{2}+\|\phi-\psi\|_{L^{2}([-1,0] ; H)}^{2}\right) \\
& =L_{\sigma}\left\|\left(\phi_{0}, \phi\right)^{T}-\left(\psi_{0}, \psi\right)^{T}\right\|_{\mathcal{H}}^{2} .
\end{aligned}
$$

If condition (ii) holds, then analogously we obtain

$$
\begin{aligned}
\left\|\Sigma\left(\phi_{0}, \phi\right)-\Sigma\left(\psi_{0}, \psi\right)\right\|_{L_{0}^{2}\left(\mathcal{H}^{\tau}\right)}^{2} & \leq L_{\sigma}\left\|\left(\phi_{0}, \phi\right)^{T}-\left(\psi_{0}, \psi\right)^{T}\right\|_{\mathcal{H}}^{2} \\
& \leq \max \{1, \tau(0)\} L_{\sigma}\left\|\left(\phi_{0}, \phi\right)^{T}-\left(\psi_{0}, \psi\right)^{T}\right\|_{\mathcal{H}^{\tau}}^{2}
\end{aligned}
$$

This shows that condition (A1) is satisfied. Finally, it follows from Proposition 6.5 below that the operator $(\mathcal{A}, D(\mathcal{A}))$ satisfies condition (GDC).

We proceed to formulate our main results on invariant measures for (6.5). For this purpose we introduce the following additional condition:

(C3) For each $\left(\phi_{0}, \phi\right) \in \mathcal{H}$ there exist $M\left(\phi_{0}, \phi\right) \geq 1, \delta\left(\phi_{0}, \phi\right)>0$ and an element $\widetilde{f}\left(\phi_{0}, \phi\right) \in \mathcal{H}$ such that

$$
\left\|\mathcal{S}(t)\left(P_{1} \phi_{0}, \phi\right)-\tilde{f}\left(\phi_{0}, \phi\right)\right\|_{\mathcal{H}} \leq M\left(\phi_{0}, \phi\right) \mathrm{e}^{-t \delta\left(\phi_{0}, \phi\right)}, \quad t \geq 0 .
$$

Observe that (C3) corresponds to condition (A3), and is trivially satisfied, if $(\mathcal{S}(t))_{t \geq 0}$ is exponentially stable which is for example the case in the setting of [5, Corollary 5.9].

Introduce the subspaces

$$
\mathcal{H}_{0}:=H_{0} \times\{0\} \quad \text { and } \quad \mathcal{H}_{1}:=H_{1} \times L^{2}([-1,0] ; H)
$$

which yield an orthogonal decomposition of $\mathcal{H}$ with projection operators

$$
\begin{aligned}
& \mathcal{P}_{0}: \mathcal{H} \longrightarrow \mathcal{H}_{0}, \quad\left(\phi_{0}, \phi\right) \longmapsto\left(P_{0} \phi_{0}, 0\right), \\
& \mathcal{P}_{1}: \mathcal{H} \longrightarrow \mathcal{H}_{1}, \quad\left(\phi_{0}, \phi\right) \longmapsto\left(P_{1} \phi_{0}, \phi\right) .
\end{aligned}
$$

The following is our main result for this section, and uses that $\mathcal{A}$ satisfies (GDC), whenever $A$ does, and some additional conditions are satisfied (this technical result is proved below in Proposition 6.5).

Theorem 6.2. Suppose that conditions (C1) - (C3) hold, that $P_{1} \sigma\left(\phi_{0}, \phi\right)=0$ for all $\left(\phi_{0}, \phi\right) \in \mathcal{H}$, and that one of the following conditions are satisfied: 
(i) $G$ is bounded on $L^{2}([-1,0] ; H), G P_{1}=P_{1} G,(S(t))_{t \geq 0}$ commutes with $P_{1}$, and

$$
\alpha>1 / 2+L_{\sigma} / 2
$$

(ii) $(S(t))_{t \geq 0}$ leaves $H_{0}=\operatorname{ran}\left(P_{0}\right)$ and $H_{1}=\operatorname{ran}\left(P_{1}\right)$ invariant, $H_{0}, H_{1}$ are orthogonal, $\operatorname{ran}(G) \subset H_{1}, G P_{0}$ extends to a bounded linear operator on $L^{2}([-1,0] ; H)$, and

$$
\alpha>1 / 2+\max \{1, \tau(0)\} L_{\sigma} / 2 .
$$

Then the assertions of Theorem 4.3 and Corollary 4.4 are applicable. In particular, for each $h:=\left(\phi_{0}, \phi\right) \in L^{2}\left(\Omega, \mathcal{F}_{0}, \mathbb{P} ; \mathcal{H}\right)$ there exists an invariant measure $\pi_{\text {Law }(h)}$ for the Markov process $\left(\mathcal{X}_{t}\right)_{t \geq 0}$, and this measure satisfies $\pi_{\operatorname{Law}(h)}=\pi_{\operatorname{Law}\left(\mathcal{P}_{1} h\right)}$.

Proof. Let us first show that condition (A2) is satisfied, i.e. that $\mathcal{S}(t)$ leaves $\mathcal{H}_{1}$ invariant and $\mathcal{P}_{1} \Sigma=0$. It follows from Lemma 6.6 below that $\mathcal{P}_{1}$ commutes with the semigroup $(\mathcal{S}(t))_{t \geq 0}$. Moreover, one has

$$
\mathcal{P}_{1} \Sigma\left(\phi_{0}, \phi\right)=\left(\begin{array}{cc}
P_{1} \sigma\left(\phi_{0}, \phi\right) & 0 \\
0 & 0
\end{array}\right)=0
$$

due to $P_{1} \sigma=0$. This shows that condition (A2) is satisfied. Condition (A3) is immediate by assumption (C3) while, by virtue of Proposition 6.5, (3.13) reduces under condition (i) to

$$
\varepsilon=2\left(\alpha-\frac{1}{2}\right)-L_{\sigma}>0,
$$

and under condition (ii) to

$$
\varepsilon=2\left(\alpha-\frac{1}{2}\right)-\max \{1, \tau(0)\} L_{\sigma}>0 .
$$

Altogether we conclude that Theorems 4.3 and 4.4 apply, which proves the assertion.

Remark 6.3. Condition (ii) is slightly more restrictive on the semigroup and the projection operators than condition (i). In contrast to the latter, condition (ii) contains delay operators like point evaluations in $H_{1}$, that is $G=\delta_{-1} P_{1}$ for $\delta_{-1} \phi=\phi(-1)$ for $\phi \in W^{1,2}\left([-1,0] ; H_{1}\right)$.

\subsection{Some technical results}

Let us first provide a sufficient and easy to check condition for the operator $\mathcal{A}$ to satisfy the generalized dissipativity condition (GDC). As a first step we recall a result from [41].

Remark 6.4. (An equivalent scalar product). Let $\tau$ be defined as in (6.7) and suppose that $\eta$ has a jump at -1 . Suppose that there exists $c \in \mathbb{R}$ such that $A-c$ is dissipative. Then the Hilbert space norm defined by

$$
\left\|\left(\phi_{0}, \phi\right)\right\|_{\mathcal{H}^{\tau}}^{2}:=\left\|\phi_{0}\right\|_{H}^{2}+\int_{-1}^{0}\|\phi(s)\|_{H}^{2} \tau(s) d s
$$


is equivalent to the original one on $\mathcal{H}$. Moreover, $\mathcal{A}-\gamma I$ is dissipative for every $\gamma \geq \max \{0, c+\tau(0)\}$ with respect to this norm, i.e.

$$
\left\langle\mathcal{A}\left(\phi_{0}, \phi\right)^{T},\left(\phi_{0}, \phi\right)\right\rangle_{\mathcal{H}^{\tau}} \leq \gamma\left\|\left(\phi_{0}, \phi\right)^{T}\right\|_{\mathcal{H}^{\tau}}, \quad \text { for all }\left(\phi_{0}, \phi\right) \in D(\mathcal{A})
$$

Based on this observation we can now provide sufficient conditions for $(\mathcal{A}, D(\mathcal{A}))$ to satisfy $(\mathrm{GDC})$.

Proposition 6.5. Suppose that A satisfies (GDC) with constants $\alpha, \beta \geq 0$.

(i) If $G$ extends to a continuous linear operator on $L^{2}([-1,0] ; H)$ and $\alpha>$ $1 / 2$, then $\mathcal{A}$ satisfies $(G D C)$, i.e.,

$$
\left\langle\mathcal{A}\left(\phi_{0}, \phi\right)^{T},\left(\phi_{0}, \phi\right)^{T}\right\rangle_{\mathcal{H}} \leq-\widetilde{\alpha}\left\|\left(\phi_{0}, \phi\right)\right\|_{\mathcal{H}}^{2}+(\widetilde{\alpha}+\widetilde{\beta})\left\|\mathcal{P}_{1}\left(\phi_{0}, \phi\right)^{T}\right\|_{\mathcal{H}}^{2},
$$

where

$$
\widetilde{\alpha}:=\alpha-\frac{1+\varepsilon^{2}}{2} \quad \text { and } \quad \widetilde{\beta}:=\beta+\alpha+\frac{1}{2 \varepsilon^{2}}\|G\|_{L\left(L^{2}([-1,0] ; H)\right)}^{2}+\frac{\varepsilon^{2}}{2},
$$

and $\varepsilon>0$ is such that $\varepsilon<\sqrt{2 \alpha-1}$.

(ii) Assume that $H_{0}, H_{1}$ provide an orthogonal decomposition of $H$ such the semigroup $(S(t))_{t \geq 0}$ generated by $(A, D(A))$ leaves $H_{0}$ and $H_{1}$ invariant. Moreover, suppose that $\operatorname{ran}(G) \subseteq H_{1}$, and that $W^{1,2}([-1,0] ; H) \ni \phi \mapsto$ $G P_{0} \phi \in H_{1}$ extends to a continuous linear operator $G P_{0}: L^{2}([-1,0] ; H) \rightarrow$ $H_{1}$ with operator norm denoted by $\left\|G P_{0}\right\|$. Define an equivalent Hilbert space norm by (6.6). If $\alpha>1 / 2$, then $\mathcal{A}$ satisfies (GDC) with respect to this norm, i.e., it holds that

$$
\begin{aligned}
\left\langle\mathcal{A}\left(\phi_{0}, \phi\right)^{T},\left(\phi_{0}, \phi\right)^{T}\right\rangle_{\mathcal{H}^{\tau}} \leq & -\left(\alpha-\frac{1}{2}\right)\left\|\left(\phi_{0}, \phi\right)\right\|_{\mathcal{H}^{\tau}} \\
& +\left(\left(\alpha-\frac{1}{2}\right)+\beta+\tau(0)+\frac{\left\|G P_{0}\right\|}{2}\right)\left\|\mathcal{P}_{1}\left(\phi_{0}, \phi\right)\right\|_{\mathcal{H}^{\tau}}^{2}
\end{aligned}
$$

Proof. (i) For $\left(\phi_{0}, \phi\right)^{T} \in D\left(\mathcal{A}_{0}\right)$ we have

$$
\begin{aligned}
\left\langle\mathcal{A}_{0}\left(\phi_{0}, \phi\right)^{T},\left(\phi_{0}, \phi\right)^{T}\right\rangle_{\mathcal{H}} & =\left\langle A \phi_{0}, \phi_{0}\right\rangle_{H}+\int_{-1}^{0}\left\langle\frac{d}{d s} \phi(s), \phi(s)\right\rangle_{H} d s \\
& =\left\langle A \phi_{0}, \phi_{0}\right\rangle_{H}+\int_{-1}^{0} \frac{1}{2} \frac{d}{d s}\|\phi(s)\|_{H}^{2} d s \\
& =\left\langle A \phi_{0}, \phi_{0}\right\rangle_{H}+\frac{1}{2}\left(\|\phi(0)\|_{H}^{2}-\|\phi(-1)\|_{H}^{2}\right) \\
& \leq\left\langle A \phi_{0}, \phi_{0}\right\rangle_{H}+\frac{1}{2}\left\|\phi_{0}\right\|_{H}^{2},
\end{aligned}
$$

where we used the fact that $\phi_{0}=\phi(0)$. Making further use of the fact that $A$ satisfies (GDC) we find

$$
\begin{gathered}
\left\langle\mathcal{A}_{0}\left(\phi_{0}, \phi\right)^{T},\left(\phi_{0}, \phi\right)^{T}\right\rangle_{\mathcal{H}} \leq-\left(\alpha-\frac{1}{2}\right)\left\|\phi_{0}\right\|_{H}^{2}+(\beta+\alpha)\left\|P_{1} \phi_{0}\right\|_{H}^{2} \\
\leq-\left(\alpha-\frac{1}{2}\right)\left\|\left(\phi_{0}, \phi\right)\right\|_{\mathcal{H}}^{2}+\left(\beta+2 \alpha-\frac{1}{2}\right)\left\|\left(P_{1} \phi_{0}, \phi\right)^{T}\right\|_{\mathcal{H}}^{2} .
\end{gathered}
$$


To estimate the operator $\mathcal{A}$ we will use that

$$
\begin{aligned}
\left\langle G \phi, \phi_{0}\right\rangle_{H} & \leq\|G \phi\|_{H}\left\|\phi_{0}\right\|_{H} \\
& \leq \frac{1}{2 \varepsilon^{2}}\|G \phi\|_{H}^{2}+\frac{\varepsilon^{2}}{2}\left\|\phi_{0}\right\|_{H}^{2} \\
& =\frac{1}{2 \varepsilon^{2}}\|G\|_{L\left(L^{2}([-1,0] ; H)\right)}^{2}\|\phi\|_{L^{2}([-1,0] ; H)}^{2}+\frac{\varepsilon^{2}}{2}\left\|\phi_{0}\right\|_{H}^{2} \\
& \leq \frac{1}{2 \varepsilon^{2}}\|G\|_{L\left(L^{2}([-1,0] ; H)\right)}^{2}\left\|\mathcal{P}_{1}\left(\phi_{0}, \phi\right)^{T}\right\|_{\mathcal{H}}^{2}+\frac{\varepsilon^{2}}{2}\left\|\left(\phi_{0}, \phi\right)^{T}\right\|_{\mathcal{H}}^{2}
\end{aligned}
$$

where $\varepsilon>0$. Thus we obtain

$$
\begin{aligned}
\left\langle\mathcal{A}\left(\phi_{0}, \phi\right)^{T},\left(\phi_{0}, \phi\right)^{T}\right\rangle_{\mathcal{H}} \\
=\left\langle\mathcal{A}_{0}\left(\phi_{0}, \phi\right)^{T},\left(\phi_{0}, \phi\right)^{T}\right\rangle_{\mathcal{H}}+\left\langle G \phi, \phi_{0}\right\rangle_{H} \\
\leq-\left(\alpha-\frac{1}{2}\right)\left\|\left(\phi_{0}, \phi\right)\right\|_{\mathcal{H}}^{2}+\left(\beta+2 \alpha-\frac{1}{2}\right)\left\|\mathcal{P}_{1}\left(\phi_{0}, \phi\right)^{T}\right\|_{\mathcal{H}}^{2} \\
\quad+\frac{1}{2 \varepsilon^{2}}\|G\|_{L\left(L^{2}([-1,0] ; H)\right)}^{2}\left\|\mathcal{P}_{1}\left(\phi_{0}, \phi\right)^{T}\right\|_{\mathcal{H}}^{2}+\frac{\varepsilon^{2}}{2}\left\|\left(\phi_{0}, \phi\right)^{T}\right\|_{\mathcal{H}}^{2} \\
=-\left(\alpha-\frac{1+\varepsilon^{2}}{2}\right)\left\|\left(\phi_{0}, \phi\right)\right\|_{\mathcal{H}}^{2}+\left(\beta+2 \alpha-\frac{1}{2}+\frac{1}{2 \varepsilon^{2}}\|G\|_{\left.L\left(L^{2}([-1,0] ; H)\right)\right)}^{2}\right) \\
\quad\left\|\mathcal{P}_{1}\left(\phi_{0}, \phi\right)^{T}\right\|_{\mathcal{H}}^{2} .
\end{aligned}
$$

Assuming $\varepsilon$ is so small that $\varepsilon<\sqrt{2 \alpha-1}$, we obtain $\alpha-\frac{1+\varepsilon^{2}}{2}>0$ and

$$
\begin{aligned}
\beta & +2 \alpha-\frac{1}{2}+\frac{1}{2 \varepsilon^{2}}\|G\|_{L\left(L^{2}([-1,0] ; H)\right)}^{2} \\
& =\left(\alpha-\frac{1+\varepsilon^{2}}{2}\right)+\beta+\alpha+\frac{1}{2 \varepsilon^{2}}\|G\|_{L\left(L^{2}([-1,0] ; H)\right)}^{2}+\frac{\varepsilon^{2}}{2}>0
\end{aligned}
$$

which proves the assertion.

(ii) As $P_{0}, P_{1}$ are complementary self-adjoint projections, they induce an orthogonal decomposition $H=H_{0} \oplus H_{1}$. Thus, for $\left(\phi_{0}, \phi\right) \in \mathcal{H}$ we have $\left(\phi_{0}, \phi\right)=\left(P_{0} \phi_{0}, P_{0} \phi\right)+\left(P_{1} \phi_{0}, P_{1} \phi\right)$ which gives also an orthogonal decomposition

$$
\mathcal{H}=\left(H_{0} \times L^{2}\left([-1,0] ; H_{0}\right)\right) \oplus\left(H_{1} \times L^{2}\left([-1,0] ; H_{1}\right)\right) .
$$

Applying Remark 6.4 to the Hilbert space $H_{1} \times L^{2}\left([-1,0] ; H_{1}\right)$ we find that

$$
\left\|P_{1} \phi_{0}\right\|_{H^{2}}+\int_{-1}^{0}\left\|P_{1} \phi(s)\right\|_{H}^{2} \tau(s) d s
$$

gives rise to a norm on $H_{1} \times L^{2}\left([-1,0] ; H_{1}\right)$ which is equivalent to the one given by $(6.2)$ when applied to $\left(P_{1} \phi_{0}, P_{1} \phi\right)$. Thus, the norm defined in (6.6) is, indeed, equivalent to the original norm on $\mathcal{H}$. Let $\left(\phi_{0}, \phi\right) \in D(\mathcal{A})$, so that 
$\phi(0)=\phi_{0}$ and we can write:

$$
\begin{aligned}
\left\langle\mathcal{A}\left(\phi_{0}, \phi\right)^{T},\left(\phi_{0}, \phi\right)^{T}\right\rangle_{\mathcal{H}^{\tau}} & \left\langle\left(A \phi_{0}+G \phi, \frac{d}{d s} \phi\right)^{T},\left(\phi_{0}, \phi\right)^{T}\right\rangle_{\mathcal{H}^{\tau}} \\
= & \left\langle A \phi_{0}, \phi_{0}\right\rangle_{H}+\left\langle G \phi, \phi_{0}\right\rangle_{H} \\
= & +\int_{-1}^{0}\left\langle\frac{d}{d s} P_{0} \phi(s), P_{0} \phi(s)\right\rangle_{H} d s+\int_{-1}^{0}\left\langle\frac{d}{d s} P_{1} \phi(s), P_{1} \phi(s)\right\rangle_{H} \tau(s) d s \\
= & I_{1}+I_{2}+I_{3}+I_{4} .
\end{aligned}
$$

For the first term $I_{1}$ we use $\phi_{0}=P_{0} \phi_{0}+P_{1} \phi_{0}$, then the fact that $P_{0}, P_{1}$ are self-adjoint projection operators and finally $P_{0} A=A P_{0}, P_{1} A=A P_{1}$ on $D(A)$ (similarly to the proof of Proposition 3.1) to find that

$$
\begin{aligned}
I_{1} & =\left\langle P_{0} A \phi_{0}, P_{0} \phi_{0}\right\rangle_{H}+\left\langle P_{1} A \phi_{0}, P_{1} \phi_{0}\right\rangle_{H} \\
& =\left\langle A P_{0} \phi_{0}, P_{0} \phi_{0}\right\rangle_{H}+\left\langle A P_{1} \phi_{0}, P_{1} \phi_{0}\right\rangle_{H} \leq-\alpha\left\|P_{0} \phi_{0}\right\|_{H}^{2}+\left\langle A P_{1} \phi_{0}, P_{1} \phi_{0}\right\rangle_{H},
\end{aligned}
$$

where the last inequality follows from (GDC) combined with $P_{1} P_{0} \phi_{0}=0$. Likewise, for the second term we use that $\operatorname{ran}(G) \subset H_{1}$ so that $P_{0} G=0$ to obtain

$$
\begin{aligned}
I_{2} & =\left\langle G \phi, P_{1} \phi_{0}\right\rangle_{H} \\
& =\left\langle G P_{0} \phi, P_{1} \phi_{0}\right\rangle_{H}+\left\langle G P_{1} \phi, P_{1} \phi_{0}\right\rangle_{H} \\
& \leq\left\|G P_{0}\right\|\left\|P_{0} \phi\right\|_{L^{2}([-1,0] ; H)}\left\|P_{1} \phi_{0}\right\|_{H}+\left\langle G P_{1} \phi, P_{1} \phi_{0}\right\rangle_{H} \\
& \leq \frac{\left\|G P_{0}\right\|}{2}\left\|P_{0} \phi\right\|_{L^{2}([-1,0] ; H)}^{2}+\frac{\left\|G P_{0}\right\|}{2}\left\|P_{1} \phi_{0}\right\|_{H}^{2}+\left\langle G P_{1} \phi, P_{1} \phi_{0}\right\rangle_{H} .
\end{aligned}
$$

For the third term $I_{3}$ we obtain

$$
I_{3}=\frac{1}{2} \int_{-1}^{0} \frac{d}{d s}\left\|P_{0} \phi(s)\right\|_{H}^{2} d s \leq \frac{1}{2}\left\|P_{0} \phi_{0}\right\|_{H}^{2} .
$$

To summarize, we obtain 


$$
\begin{aligned}
&\left\langle\mathcal{A}\left(\phi_{0}, \phi\right)^{T},\left(\phi_{0}, \phi\right)^{T}\right\rangle_{\mathcal{H}^{\tau}} \\
& \leq-\left(\alpha-\frac{1}{2}\right)\left\|P_{0} \phi_{0}\right\|_{H}^{2}+\frac{\left\|G P_{0}\right\|}{2}\left(\left\|P_{1} \phi_{0}\right\|_{H}^{2}+\left\|P_{0} \phi\right\|_{L^{2}([-1,0] ; H)}^{2}\right) \\
&+\left\langle A P_{1} \phi_{0}, P_{1} \phi_{0}\right\rangle_{H}+\left\langle G P_{1} \phi, P_{1} \phi_{0}\right\rangle_{H}+\int_{-1}^{0}\left\langle\frac{d}{d s} P_{1} \phi(s), P_{1} \phi(s)\right\rangle_{H} \tau(s) d s \\
&=-\left(\alpha-\frac{1}{2}\right)\left\|\left(\phi_{0}, \phi\right)^{T}\right\|_{\mathcal{H}^{\tau}}^{2}+\left(\alpha-\frac{1}{2}\right)\left\|P_{1} \phi_{0}\right\|_{H}^{2}+\left(\alpha-\frac{1}{2}\right) \int_{-1}^{0}\left\|P_{0} \phi(s)\right\|_{H}^{2} d s \\
&+\left(\alpha-\frac{1}{2}\right) \int_{-1}^{0}\left\|P_{1} \phi(s)\right\|_{H}^{2} \tau(s) d s+\frac{\left\|G P_{0}\right\|_{\|}}{2}\left\|P_{1} \phi_{0}\right\|_{H}^{2}+\frac{\left\|G P_{0}\right\|}{2} \int_{-1}^{0}\left\|P_{0} \phi(s)\right\|_{H}^{2} d s \\
&+\left\langle\mathcal{A}\left(P_{1} \phi_{0}, P_{1} \phi\right)^{T},\left(P_{1} \phi_{0}, P_{1} \phi\right)^{T}\right\rangle_{\mathcal{H}^{\tau}} \\
& \leq-\left(\alpha-\frac{1}{2}\right)\left\|\left(\phi_{0}, \phi\right)^{T}\right\|_{\mathcal{H}^{\tau}}^{2}+\left(\alpha-\frac{1}{2}+\gamma+\frac{\left\|G P_{0}\right\|}{2}\right)\left\|P_{1} \phi_{0}\right\|_{H}^{2} \\
&+\left(\alpha-\frac{1}{2}+\frac{\left\|G P_{0}\right\|}{2}\right) \int_{-1}^{0}\left\|P_{0} \phi(s)\right\|_{H}^{2} d s+\left(\alpha-\frac{1}{2}+\gamma\right) \int_{-1}^{0}\left\|P_{1} \phi(s)\right\|_{H}^{2} \tau(s) d s \\
& \leq-\left(\alpha-\frac{1}{2}\right)\left\|\left(\phi_{0}, \phi\right)^{T}\right\|_{\mathcal{H}^{\tau}}^{2}+\left(\alpha-\frac{1}{2}+\gamma+\frac{\left\|G P_{0}\right\|}{2}\right)\left\|\mathcal{P}_{1}\left(\phi_{0}, \phi\right)^{T}\right\|_{\mathcal{H}^{\tau}}^{2},
\end{aligned}
$$

where we have used the fact that $A-\beta I$ is dissipative so that by Remark 6.4 with $\gamma=\beta+\tau(0)$

$$
\begin{aligned}
\left\langle\mathcal{A}\left(P_{1} \phi_{0}, P_{1} \phi\right)^{T},\left(P_{1} \phi_{0}, P_{1} \phi\right)^{T}\right\rangle_{\mathcal{H}^{\tau}} & \leq \gamma\left\|\left(P_{1} \phi_{0}, P_{1} \phi\right)^{T}\right\|_{\mathcal{H}^{\tau}}^{2} \\
& =\gamma\left\|P_{1} \phi_{0}\right\|_{H}^{2}+\gamma \int_{-1}^{0}\left\|P_{1} \phi(s)\right\|_{H}^{2} \tau(s) d s .
\end{aligned}
$$

This proves the assertion.

The next result has been used in the previous proof.

Lemma 6.6. Consider the setting of stochastic delay equation, i.e., let $\left(\mathcal{S}_{0}(t)\right)_{t \geq 0}$, $(\mathcal{S}(t))_{t \geq 0},\left(\mathcal{A}_{0}, D\left(\mathcal{A}_{0}\right)\right),(\mathcal{A}, D(\mathcal{A})), G, P_{1}$ as in Sects. 6.1, 6.2 and Theorem 6.2. In particular suppose that $P_{1} S(t)=P_{1} S(t)$, where $S(t)$ is given in (6.3). Then

$$
\mathcal{P}_{1} \mathcal{S}(t)=\mathcal{S}(t) \mathcal{P}_{1}, \quad t \geq 0
$$

Proof. Let us first consider the case where $G$ satisfies assumption (i) from Proposition 6.5, i.e. $G$ is bounded from $L^{2}([-1,0] ; H)$ to $H$. Since $G$ is bounded we obtain from the bounded perturbation theorem (the Dyson-Phillips series) the representation

$$
\mathcal{S}(t)=\sum_{n=0}^{\infty} \mathcal{S}_{0}^{(n)}(t)
$$

where the series converges in $L(\mathcal{H})$, and $\mathcal{S}_{0}^{(n)}(t)$ is inductively defined by

$$
\mathcal{S}_{0}^{(0)}(t)=\mathcal{S}_{0}(t), \quad \mathcal{S}_{0}^{(n+1)}(t)=\int_{0}^{t} \mathcal{S}_{0}^{(n)}(s)\left(\begin{array}{cc}
0 & G \\
0 & 0
\end{array}\right) \mathcal{S}_{0}(t-s) d s .
$$


Thus it suffices to prove that

$$
\mathcal{P}_{1} \mathcal{S}_{0}^{(n)}(t)=\mathcal{S}_{0}^{(n)}(t) \mathcal{P}_{1}, \quad n \geq 1, \quad t \geq 0 .
$$

For $n=0$ we use the particular form of $\mathcal{P}_{1}$ and $\mathcal{S}_{0}(t)$ to find that

$$
\begin{aligned}
\mathcal{P}_{1} \mathcal{S}_{0}(t)\left(\phi_{0}, \phi\right)^{T} & =\mathcal{P}_{1}\left(\begin{array}{c}
S(t) \phi_{0} \\
S_{t} \phi_{0}+T_{0}(t) \phi
\end{array}\right) \\
& =\left(\begin{array}{c}
P_{1} S(t) \phi_{0} \\
S_{t} \phi_{0}+T_{0}(t) \phi
\end{array}\right)=\left(\begin{array}{c}
S(t) P_{1} \phi_{0} \\
S_{t} \phi_{0}+T_{0}(t) \phi
\end{array}\right)=\mathcal{S}_{0}(t) \mathcal{P}_{1}\left(\phi_{0}, \phi\right)^{T},
\end{aligned}
$$

where we have used the assumption that $S(t)$ commutes with $P_{1}$. Now suppose that (6.8) holds for some $n \geq 0$. Then

$$
\begin{aligned}
\mathcal{P}_{1} \mathcal{S}_{0}^{(n+1)}(t) & =\mathcal{P}_{1} \mathcal{S}_{0}(t)+\int_{0}^{t} \mathcal{P}_{1} \mathcal{S}_{0}^{(n)}(s)\left(\begin{array}{cc}
0 & G \\
0 & 0
\end{array}\right) \mathcal{S}_{0}(t-s) d s \\
& =\mathcal{S}_{0}(t) \mathcal{P}_{1}+\int_{0}^{t} \mathcal{S}_{0}^{(n)}(s)\left(\begin{array}{cc}
0 & G \\
0 & 0
\end{array}\right) \mathcal{S}_{0}(t-s) \mathcal{P}_{1} d s=\mathcal{S}_{0}^{(n+1)}(t) \mathcal{P}_{1}
\end{aligned}
$$

where we have used that

$$
\begin{aligned}
\mathcal{P}_{1}\left(\begin{array}{cc}
0 & G \\
0 & 0
\end{array}\right)\left(\phi_{0}, \phi\right)^{T} & =\mathcal{P}_{1}(G \phi, 0)^{T} \\
& =\left(P_{1} G \phi, 0\right)=\left(G P_{1} \phi, 0\right)=\left(\begin{array}{cc}
G & 0 \\
0 & 0
\end{array}\right) \mathcal{P}_{1}\left(\phi_{0}, \phi\right)^{T}
\end{aligned}
$$

This completes the proof for the case where $G: L^{2}([-1,0] ; H) \longrightarrow H$ is bounded.

Let us now consider the case where condition (ii) from Proposition 6.5 holds. Following [5, Theorem 3.29] we know that the semigroup $(\mathcal{S}(t))_{t \geq 0}$ is constructed as a Miyadera-Voigt perturbation and hence has due to [14, Chapter III, Corollary 3.15] a series representation of the form

$$
\mathcal{S}(t)=\sum_{n=0}^{\infty} \bar{V}^{n} \mathcal{S}_{0}(t),
$$

where $\bar{V}$ denotes the closure of the operator

$$
F \longmapsto V F(t):=\int_{0}^{t} F(s)\left(\begin{array}{cc}
0 & G \\
0 & 0
\end{array}\right) \mathcal{S}_{0}(t-s) d s,
$$

where $F \in C\left(\left[0, t_{0}\right] ; L_{s}(\mathcal{H})\right)$ (for some small but fixed $t_{0}>0$ ) and $L_{s}(\mathcal{H})$ denotes the space of bounded linear operators over $\mathcal{H}$ equipped with the strong operator topology. Following the same computations as in the first case, we can prove that $\mathcal{P}_{1} V^{n} \mathcal{S}_{0}(t)=V^{n} \mathcal{S}_{0}(t) \mathcal{P}_{1}$ and hence $\mathcal{P}_{1} \bar{V}^{n} \mathcal{S}_{0}(t)=\bar{V}^{n} \mathcal{S}_{0}(t) \mathcal{P}_{1}$. This proves the assertion also in this case.

\section{Acknowledgements}

Dennis Schroers was funded within the project STORM: Stochastics for TimeSpace Risk Models, from the Research Council of Norway (RCN). Project Number: 274410. 
Funding Open Access funding enabled and organized by Projekt DEAL.

Open Access. This article is licensed under a Creative Commons Attribution 4.0 International License, which permits use, sharing, adaptation, distribution and reproduction in any medium or format, as long as you give appropriate credit to the original author(s) and the source, provide a link to the Creative Commons licence, and indicate if changes were made. The images or other third party material in this article are included in the article's Creative Commons licence, unless indicated otherwise in a credit line to the material. If material is not included in the article's Creative Commons licence and your intended use is not permitted by statutory regulation or exceeds the permitted use, you will need to obtain permission directly from the copyright holder. To view a copy of this licence, visit http://creativecommons. org/licenses/by/4.0/.

Publisher's Note Springer Nature remains neutral with regard to jurisdictional claims in published maps and institutional affiliations.

\section{Appendix A: Itô formula}

Below we recall an Itô formula for Hilbert space valued semimartingales of the form

$$
X(t)=X(0)+\int_{0}^{t} a(s) d s+\int_{0}^{t} \sigma(s) d W_{s}+\int_{0}^{t} \int_{E} \gamma(s, \nu) \tilde{N}(d s, d \nu)
$$

where $a$ and $\sigma$ are as before and $(\gamma(t, \nu))_{t \geq 0}$ is a predictable, $H$-valued stochastic process for each $\nu \in E$ such that

$$
\mathbb{E}\left[\int_{0}^{t} \int_{E}\|\gamma(s, \nu)\|_{H}^{2} \mu(d \nu) d s\right]<\infty
$$

and

$$
\mathbb{E}\left[\int_{0}^{t}\|\sigma(s)\|_{L_{2}^{0}}^{2} d s\right]<\infty .
$$

For this purpose we first introduce the class of quasi-sublinear functions.

Definition A.1. (Sublinear Functions) A continuous, non-decreasing function $h: \mathbb{R}_{+} \rightarrow \mathbb{R}_{+}$is called quasi-sublinear, if there exists a constant $C>0$ such that

$$
\begin{aligned}
h(x+y) & \leq C(h(x)+h(y)) \\
h(x y) & \leq C(h(x) h(y))
\end{aligned}
$$

for all $x, y \geq 0$.

The following Itô-Formula is a combination of [20,29] (see also [28]).

Theorem A.2. (Generalized Itô-Formula). Let $F \in C^{2}\left(\mathbb{R}_{+} \times H, \mathbb{R}\right)$ and suppose there exist quasi-sublinear functions $h_{1}, h_{2}: \mathbb{R}_{+} \rightarrow \mathbb{R}_{+}$such that for all $t \geq 0$ and $x \in H$

$$
\left\|F_{x}(t, x)\right\|_{H} \leq h_{1}\left(\|x\|_{H}\right), \quad\left\|F_{x x}(t, x)\right\|_{L(H, L(H, \mathbb{R}))} \leq h_{2}\left(\|x\|_{H}\right)
$$


and

$$
\begin{aligned}
\int_{0}^{t} & \int_{E}\|\gamma(s, \nu)\|_{H}^{2} \mu(d \nu) d s+\int_{0}^{t} \int_{E} h_{1}\left(\|\gamma(s, \nu)\|_{H}\right)^{2}\|\gamma(s, \nu)\|_{H}^{2} \mu(d \nu) d s \\
& +\int_{0}^{t} \int_{E} h_{2}\left(\|\gamma(s, \nu)\|_{H}\right)\|\gamma(s, \nu)\|_{H}^{2} \mu(d \nu) d s<\infty
\end{aligned}
$$

Then $\mathbb{P}$-almost surely for each $t \geq 0$ :

$$
\begin{aligned}
& \int_{0}^{t}\left\|F_{t}(s, X(s))\right\|_{H} d s+\int_{0}^{t} \int_{E}|F(s, X(s)+\gamma(s, \nu))-F(s, X(s))|^{2} \mu(d \nu) d s \\
& \quad+\int_{0}^{t} \int_{E} \mid F(s, X(s)+\gamma(s, \nu))-F(s, X(s)) \\
& \left.\quad-\left\langle F_{x}(s, X(s)), \gamma(s, \nu)\right\rangle_{H}\right) \mid \mu(d \nu) d s<\infty .
\end{aligned}
$$

Moreover, the generalized Itô-formula holds $\mathbb{P}$-almost surely for each $t \geq 0$ and

$$
\begin{aligned}
F(t, X(t))= & F(0, X(0))+\int_{0}^{t} \mathcal{L} F(s, X(s)) d s+\int_{0}^{t}\left\langle F_{x}(s, X(s)), \sigma(s) d W_{s}\right\rangle_{H} \\
& +\int_{0}^{t+} \int_{E}\{F(s, X(s-)+\gamma(s, \nu))-F(s, X(s-))\} \tilde{N}(d s, d \nu)
\end{aligned}
$$

where $\mathcal{L} F(x, X(s))$ is given by

$$
\begin{aligned}
\mathcal{L} F(s, X(s))= & \int_{0}^{t}\left\{F_{t}(s, X(s))+\left\langle F_{x}(s, X(s)), a(s)\right\rangle_{H}\right\} d s \\
& +\frac{1}{2} \int_{0}^{t} \operatorname{tr}\left[F_{x x}(s, X(s)) \sigma(s) Q \sigma(s)^{*}\right] d s \\
& +\int_{0}^{t} \int_{E}\{F(s, X(s)+\gamma(s, \nu))-F(s, X(s)) \\
& \left.-\left\langle F_{x}(s, X(s)), \gamma(s, \nu)\right\rangle_{H}\right\} \mu(d \nu) d s
\end{aligned}
$$

\section{References}

[1] Albeverio, S., Mandrekar, V., Rüdiger, B.: Existence of mild solutions for stochastic differential equations and semilinear equations with non-gaussian lévy noise. Stoch. Process. Appl. 119(3), 835-863 (2009)

[2] Albeverio, S., Gawarecki, L., Mandrekar, V., Rüdiger, B., Sarkar, B.: Itô formula for mild solutions of SPDEs with Gaussian and non-Gaussian noise and applications to stability properties. Random Oper. Stoch. Equ. 25(2), 79-105 (2017)

[3] Applebaum, D.: Infinite dimensional Ornstein-Uhlenbeck processes driven by Lévy processes. Probab. Surv. 12, 33-54 (2015)

[4] Barbu, V., Röckner, M., Zhang, D.: Stochastic nonlinear Schrödinger equations. Nonlinear Anal. 136, 168-194 (2016) 
[5] Bátkai, A., Piazzera, S.: Semigroups for Delay Equations, Research Notes in Mathematics, vol. 10. A K Peters Ltd, Wellesley (2005)

[6] Benth, F.E., Detering, N., Kruehner, P.: Stochastic Volterra integral equations and a class of first order stochastic partial differential equations (2019). arXiv:1903.05045

[7] Butkovsky, O.: Subgeometric rates of convergence of Markov processes in the Wasserstein metric. Ann. Appl. Probab. 24(2), 526-552 (2014)

[8] Cox, S., Górajski, M.: Vector-valued stochastic delay equations - a semigroup approach. Semigroup Forum 82(3), 389-411 (2011)

[9] Da Prato, G., Debussche, A.: Ergodicity for the 3D stochastic Navier-Stokes equations. J. Math. Pures Appl. (9) 82(8), 877-947 (2003)

[10] Da Prato, G., Zabczyk, J.: Stochastic Equations in Infinite Dimensions. Encyclopedia of Mathematics and Its Applications. Cambridge University Press, Cambridge (2014)

[11] Filipović, S.T.D., Teichmann, J.: Term structure models driven by Wiener processes and poisson measures: existence and positivity. SIAM J. Financ. Math. $\mathbf{1}(1), 523-554$ (2010)

[12] Dawson, D.A., Etheridge, A.M., Fleischmann, K., Mytnik, L., Perkins, E.A., Xiong, J.: Mutually catalytic branching in the plane: infinite measure states. Electron. J. Probab. 7(15), 61 (2002)

[13] de Bouard, A., Debussche, A.: The stochastic nonlinear Schrödinger equation in $H^{1}$. Stoch. Anal. Appl. 21(1), 97-126 (2003)

[14] Engel, K.J., Brendle, S., Nagel, R., Campiti, M., Hahn, T., Metafune, G., Nickel, G., Pallara, D., Perazzoli, C., Rhandi, A., et al.: One-Parameter Semigroups for Linear Evolution Equations. Graduate Texts in Mathematics. Springer, New York (1999)

[15] Filipović, D.: Consistency Problems for HJM Interest Rate Models. Springer, Berlin (2001)

[16] Filipović, D.: Models, Term-Structure: A Graduate Course. Springer Finance. Springer, Berlin (2009)

[17] Filipović, D., Tappe, S., Teichmann, J.: Jump-diffusions in Hilbert spaces: existence, stability and numerics. Stochastics 82(5), 475-520 (2010)

[18] Flandoli, F.: Dissipativity and invariant measures for stochastic Navier-Stokes equations. NoDEA Nonlinear Differ. Equ. Appl. 1(4), 403-423 (1994)

[19] Friesen, M., Jin, P., Rüdiger, B.: Stochastic equation and exponential ergodicity in Wasserstein distances for affine processes. Ann. Appl. Prob. (to appear) (2020)

[20] Gawarecki, L., Mandrekar, V.: Stochastic Differential Equations in Infinite Dimensions: with Applications to Stochastic Partial Differential Equations. Probability and Its Applications. Springer, Berlin (2010) 
[21] Hairer, M., Mattingly, J.C., Scheutzow, M.: Asymptotic coupling and a general form of Harris' theorem with applications to stochastic delay equations. Probab. Theory Relat. Fields 149(1-2), 223-259 (2011)

[22] Hairer, M., Mattingly, J.C.: Ergodicity of the 2D Navier-Stokes equations with degenerate stochastic forcing. Ann. Math. (2) 164(3), 993-1032 (2006)

[23] Hairer, M., Mattingly, J.C.: Yet Another Look at Harris' Ergodic Theorem for Markov Chains, Seminar on Stochastic Analysis, Random Fields and Applications VI, Progr. Probab., vol. 63, pp. 109-117. Birkhäuser/Springer, Basel (2011)

[24] Heath, D., Jarrow, R., Morton, A.: Bond pricing and the term structure of interest rates: a new methodology for contingent claims valuation. Econometrica 60, 77-105 (1992)

[25] Kulik, A., Scheutzow, M.: Generalized couplings and convergence of transition probabilities. Probab. Theory Relat. Fields 171(1-2), 333-376 (2018)

[26] Kurtz, T.G.: The Yamada-Watanabe-Engelbert theorem for general stochastic equations and inequalities. Electron. J. Probab. 12, 951-965 (2007)

[27] Linde, W.: Probability in Banach Spaces-Stable and Infinitely Divisible Distributions. A Wiley-Interscience Publication, 2nd edn. Wiley, Chichester (1986)

[28] Mandrekar, V., Rüdiger, B.: Stochastic Integration in Banach Spaces: Theory and Applications (Probability Theory and Stochastic Modelling Book 73). Springer (2014)

[29] Mandrekar, V., Rüdiger, B., Tappe, S.: Itô's Formula for Banach-Space-Valued Jump Processes Driven by Poisson Random Measures. Seminar on Stochastic Analysis, Random Fields and Applications VII, pp. 171-186. Springer, Basel (2013)

[30] Mytnik, L.: Uniqueness for a mutually catalytic branching model. Probab. Theory Relat. Fields 112(2), 245-253 (1998)

[31] Pazy, A.: Semigroups of Linear Operators and Applications to Partial Differential Equations, Applied Mathematical Sciences, vol. 44. Springer, New York (1983)

[32] Peszat, S., Zabczyk, J.: Stochastic Partial Differential Equations with Lévy Noise: An Evolution Equation Approach. Encyclopedia of Mathematics and Its Applications. Cambridge University Press, Cambridge (2007)

[33] Priola, E., Shirikyan, A., Lihu, X., Zabczyk, J.: Exponential ergodicity and regularity for equations with Lévy noise. Stoch. Process. Appl. 122(1), 106-133 (2012)

[34] Priola, E., Lihu, X., Zabczyk, J.: Exponential mixing for some SPDEs with Lévy noise. Stoch. Dyn. 11(2-3), 521-534 (2011)

[35] Priola, E., Zabczyk, J.: On Linear Evolution Equations for a Class of Cylindrical Lévy Noises, Stochastic Partial Differential Equations and Applications, Quad. Mat., vol. 25, pp. 223-242. Dept. Math., Seconda Univ. Napoli, Caserta (2010) 
[36] Rusinek, A.: Mean reversion for HJMM forward rate models. Adv. Appl. Probab. 42(2), 371-391 (2010)

[37] Sritharan, S.S., Sundar, P.: Large deviations for the two-dimensional NavierStokes equations with multiplicative noise. Stoch. Process. Appl. 116(11), 16361659 (2006)

[38] Tehranchi, M.: A note on invariant measures for HJM models. Finance Stochast. 9(3), 389-398 (2005)

[39] van Gaans, O.: Invariant measures for stochastic evolution equations with Hilbert space valued Lévy noise (2005)

[40] Villani, C.: Optimal transport, Grundlehren der Mathematischen Wissenschaften [Fundamental Principles of Mathematical Sciences], vol. 338. Springer, Berlin (2009) (Old and new)

[41] Webb, G.F.: Functional differential equations and nonlinear semigroups in $L^{p}$ spaces. J. Differ. Equ. 20(1), 71-89 (1976) 
Bálint Farkas and Barbara Rüdiger

School of Mathematics and Natural Sciences

University of Wuppertal

Gaußstraße 20

42119 Wuppertal

Germany

e-mail: ruediger@uni-wuppertal.de

Bálint Farkas

e-mail: farkas@math.uni-wuppertal.de

Martin Friesen

School of Mathematics

Dublin City University

Dublin

Ireland

e-mail: martin.friesen@dcu.ie

Dennis Schroers

Department of Mathematics

University of Oslo

Oslo

Norway

e-mail: dennissc@math.uio.no

Received: 1 August 2020.

Accepted: 16 March 2021. 